Göteborg ITP 95-20

FIAN/TD/16-95

September 1995

\title{
Generalized Toda Field Theories
}

\author{
Lars Brink \\ Institute for Theoretical Physics, S-412 96 Göteborg, Sweden
}

\begin{abstract}
Mikhail A. Vasiliev
I.E. Tamm Theoretical Department, P.N. Lebedev Physical Institute, 117924, Leninsky Prospect 53, Moscow, Russia
\end{abstract}

\begin{abstract}
In this paper we introduce a unified approach to Toda field theories which allows us to formulate the classes of $A_{n}, B_{n}$ and $C_{n}$ models as unique models involving an arbitrary continuous parameter $\nu$. For certain values of $\nu$, the model describes the standard Toda theories. For other values of $\nu$ it defines a class of models that involve infinitely many fields. These models interpolate between the various standard Toda field theories. They are conformally invariant and possess infinitely many conserved higher-spin currents thus making them candidates for a new set of integrable systems. A general construction is performed, which can effectively be used for the derivation of explicit forms of particular higher-spin currents. We also study the currents in a different representation in which they are linear in the dynamical variables having, however, a non-linear Poisson bracket algebra. An explicit formula for this Poisson structure is found.
\end{abstract}




\section{Introduction}

Toda field theory encodes a class of $2 \mathrm{~d}$ field theories which are both integrable [1, 2, 3] and conformal [4, 5, 6] and therefore quite interesting from various points of view. These models have attracted a considerable interest in recent years both from a theoretical point of view as well as from a physical one (for a review see e.g. [7] and references therein). The particular Toda models are classified according to the classical Lie algebra root systems and form thus an infinite set of models. In this paper we introduce a unified approach which allows us to formulate the classes of $A_{n}, B_{n}$ and $C_{n}$ models as unique models involving an arbitrary continuous parameter $\nu$. For $2 \nu=2 n+1$, where $n$ is a non-negative integer, the model describes the standard $A_{n}, B_{n}$ and $C_{n}$ (or $A_{n-1}, B_{n-1}$ and $C_{n-1}$ ) Toda theories. For $2 \nu \neq 2 n+1$ it defines a class of models that involve infinitely many fields. These models interpolate between the various standard (finite) Toda field theories [8]. They are also conformally invariant and possess infinitely many conserved higher-spin currents thus making them candidates for a new set of integrable systems. The limiting case $\nu \rightarrow \infty$ corresponds to the continuous Toda system [9].

The scheme we will follow is based on infinite-dimensional algebras proposed in [10] in the context of the analysis of higher-spin theories. These algebras described in Section 2 belong to a class of algebras denoted as $W_{1+\infty}$ (or wedge algebras) [11]. The proposed model turns out indeed to be deeply related to higher-spins physics. It corresponds to vacuum solutions of the class of $2 \mathrm{~d}$ higher-spin models introduced recently in [12] as well as to dimensionally reduced solutions of the $d=3$ [13, [14] and $d=4$ [15] higher-spin gauge theories. From this viewpoint the Toda systems we focus on in this paper are of interest as describing various background geometries of the higher-spin gauge models. Note that this interpretation is parallel to the interpretation of the ordinary Toda field theories as particular solutions of the $W$ - gravity models described e.g. in [16].

The unified character of our approach allows us to study properties of the model in different representations. In one such representation described in Section 3 the equivalence to the ordinary Toda field theories can be established. This is done in Section 4. The higher-spin symmetries are discussed in Section 5 and the general construction of the corresponding currents is performed in Section 6. In that section we also study the currents in a different representation in which they are linear in the dynamical variables having, however, a non-linear Poisson bracket algebra. In this representation the study of the algebra of the currents is much simplified. The form of this algebra seems to be new and is different from that of the algebra $\tilde{W}_{1+\infty}$ considered in [19]. This method can effectively be used for the derivation of explicit forms of particular local higherspin currents both in infinite and finite Toda field theories in which case the problem of constructing local higher-spin currents has been extensively studied by various methods (see e.g. [2]-[0], [17, 18]). We demonstrate this for some simplest examples in Section 7 where we also analyze conformal properties of higher-spin currents. Appendices A and $\mathrm{B}$ contain some technical details of the proofs. The main definitions and notations are collected in Appendix C.

In this paper we confine ourselves to the study of the classical models. 


\section{Algebra}

The central algebraic element of our approach is to consider a function $\phi\left(T^{0}\right)$ of the operator $T^{0}$, instead of describing dynamical fields of the Toda system $\phi^{i}$ which take their values in the Cartan subalgebra of $A_{n}$, where

$$
T^{0}=\frac{1}{4}\left\{a^{-}, a^{+}\right\} .
$$

The oscillators $a^{ \pm}$obey the commutation relation

$$
\left[a^{-}, a^{+}\right]=1+2 \nu K
$$

where $\nu$ is an arbitrary constant while the operator $K$ possesses the properties

$$
K a^{ \pm}=-a^{ \pm} K ; \quad K^{2}=1 .
$$

The crucial property of the commutation relations (2.2), (2.3) is that the operator $T^{0}$ along with the generators

$$
T^{ \pm}=\frac{1}{2}\left(a^{ \pm}\right)^{2}
$$

form $s l_{2}$ commutation relations

$$
\left[T^{0}, T^{ \pm}\right]= \pm T^{ \pm}, \quad\left[T^{-}, T^{+}\right]=2 T^{0}
$$

independently of the value of $\nu$. Note that based on the definitions (2.1)-(2.4), one can derive the following useful formulae

$$
T^{-} T^{+}=\left(T^{0}\right)^{2}+T^{0}-C, \quad T^{+} T^{-}=\left(T^{0}\right)^{2}-T^{0}-C,
$$

where

$$
C=-\frac{1}{16}\left(3+4 \nu K-4 \nu^{2}\right)
$$

is the quadratic Casimir operator of $s l_{2}$ in the realization (2.1)-(2.4). Since $K$ commutes with even combinations of the oscillators $a^{ \pm}$, it can be replaced with 1 when acting on even functions of $a^{ \pm}$(the value -1 is equivalent by $\nu \leftrightarrow-\nu$ ). As a result, this realization allows one to describe various types of representations of $s l_{2}$ corresponding to different values of the Casimir operator in a uniform way.

Let us note that the modified oscillators (2.2) have been considered in the literature in various contexts and realizations [20]. In [10] the relations (2.2), (2.3) were considered as the defining relations for an associative algebra $A q(2 ; 2 \nu)$ with the generating elements $a^{ \pm}$and $K$. This algebra can be shown [21] to coincide with the factor of the enveloping algebra of $\operatorname{ssp}(1 ; 2), U(\operatorname{osp}(1 ; 2))$, over its ideal generated by the quadratic Casimir operator by factoring out all elements of the form $\left(C_{2}-c_{2}\right) a$ where $a \in U(\operatorname{osp}(1 ; 2))$ and $c_{2}=\frac{1}{16}\left(4 \nu^{2}-1\right)$ is an arbitrary constant. The associative algebras $A q(2 ; 2 \nu)$ give rise to the Lie (super)algebras with the (super)commutator $[f, g\}$ as a product law. These algebras are known in the literature as centerless (super) $W_{\infty}(\lambda)$ [21] $\left(\lambda=\frac{1}{4}(2 \nu+1)\right)$ or wedge algebras [11]. Note that the simple components of the even subalgebra of $A q(2 ; 2 \nu)$, 
which are isomorphic to the factor algebras of $U\left(s l_{2}\right)$ over appropriate ideals generated by the quadratic Casimir operator, were studied in [22]. Infinite-dimensional algebras of this class play a fundamental role in the higher-spin gauge theories developed in [23], [13, 14, 15] (and references therein). In [14] it was argued in particular that $\nu$ can be interpreted as a vacuum value of a certain scalar field in the theory of higher-spin-matter interactions in three space-time dimensions.

A general element of $A q(2 ; 2 \nu)$ can be cast into the form

$$
B=\sum_{A=0}^{1} \sum_{n=0}^{\infty} \frac{1}{n !} b_{\alpha_{1} \ldots \alpha_{n}}^{A} K^{A} a^{\alpha_{1}} \ldots a^{\alpha_{n}},
$$

where the coefficients $b_{\alpha_{1} \ldots \alpha_{n}}^{A}$ are totally symmetric in the (spinorial) indices $\alpha_{n}(\alpha= \pm)$ which implies Weyl ordering. Note that the indices are raised and lowered by the $2 \times 2$ symplectic form $\epsilon_{\alpha \beta}=-\epsilon_{\beta \alpha}, \epsilon_{-+}=\epsilon^{-+}=1, A_{\alpha}=A^{\beta} \epsilon_{\beta \alpha}, A^{\alpha}=\epsilon^{\alpha \beta} A_{\beta}$.

In the sequel we will use the following notation for the basis elements of the algebra

$$
\left.E_{n, m}=\left(\left(a^{+}\right)^{n}\left(a^{-}\right)^{m}\right)_{W} \equiv \frac{1}{(n+m) !}\left(\left(a^{+}\right)^{n}\left(a^{-}\right)^{m}+((n+m) !-1)\right) \text { permutations }\right) \text {. }
$$

Equivalently one can say that $E_{n, m}$ is such an element $B$ (2.8) in which $b_{\alpha_{1} \ldots \alpha_{n+m}}^{A}$ is different from zero only when $A=0$ and $n$ out of $n+m$ indices $\alpha$ take the value " + " while the other $m$ indices take the value "-". We will use the notation $h_{n}$ for the gradezero elements $E_{n, n}$ as they form a basis of the Cartan-type commutative subalgebra of $A q(2 ; 2 \nu)$

$$
h_{n}=E_{n, n}
$$

Note that

$$
T^{+}=\frac{1}{2} E_{2,0}, \quad T^{-}=\frac{1}{2} E_{0,2}, \quad T^{0}=\frac{1}{2} h_{1} .
$$

It should be mentioned that there is no proper generalization of the Cartan-Chevalley basis to the full infinite-dimensional algebra $A q(2 ; 2 \nu)$. Note however that a more general concept of "continuum Lie algebras" [25] might be useful in this respect.

It is an important property of $A q(2 ; 2 \nu)$ that it possesses a supertrace operation [10], i.e. a linear complex valued function $\operatorname{str}()$ on $A q(2 ; 2 \nu)$ such that

$$
\operatorname{str}(A B)=(-1)^{\pi(A)} \operatorname{str}(B A)=(-1)^{\pi(B)} \operatorname{str}(B A),
$$

where $\pi(B)$ is defined to be equal to 0 or 1 for monomials with even or odd powers $n$ in (2.8), respectively.

In [10] it was shown that there exists a unique supertrace operation (up to a scale factor) on $A q(2 ; 2 \nu)$

$$
\operatorname{str}(B)=b^{0}-2 \nu b^{1} .
$$

The existence of a (super)trace operation is important in many respects. In particular, the (super)trace allows one to construct invariant forms on the algebra as

$$
\operatorname{str}\left(B_{1} B_{2} \ldots B_{n}\right) .
$$


As a result one can construct invariants essentially in the same way as for finitedimensional algebras with the aid of an ordinary (super)trace. From the viewpoint of field theoretical applications this gives a possibility to construct invariant Lagrangians with the field variables taking their values in $A q(2 ; 2 \nu)$. Another useful application of invariant forms is that null vectors of any invariant bilinear form span some ideal of the algebra. As a result, having a supertrace operation one has a constructive possibility to search for ideals of the algebra. Moreover, the ideals of this particular class have the very important property that they automatically decouple from the factor algebra under the supertrace operation.

In [10 an explicit formula for the bilinear invariant form induced by the supertrace (2.13) was derived. The final result is

$$
\operatorname{str}\left(B_{1} B_{2}\right)=\sum_{n=0}^{\infty} \sum_{A, B=0,1} \frac{1}{n !} \beta^{A B}(n) b_{1 \alpha_{1} \ldots \alpha_{n}}^{A} b_{2}^{B \alpha_{1} \ldots \alpha_{n}}
$$

where

$$
\begin{aligned}
\beta^{A B}(n)= & (-1)^{B n} 2^{-n} \prod_{l=0}^{[(n-1) / 2]}\left(1-\frac{4 \nu^{2}}{(2 l+1)^{2}}\right) \times \\
& {\left[\delta(A+B)-\frac{1}{2}\left(1+(-1)^{n}\right) \frac{2 \nu}{n+1} \delta(A+B+1)\right] }
\end{aligned}
$$

for $n>0$ and

$$
\beta^{A B}(0)=[\delta(A+B)-2 \nu \delta(A+B+1)] .
$$

Here $\left[\frac{m}{2}\right]$ denotes the integral part of $\frac{m}{2}\left(\left[\frac{m}{2}\right] \leq \frac{m}{2}\right)$, while $A+B$ and $A+B+1$ are assumed to take values 0 or 1 (i.e. with the convention $1+1=0$ ).

From this formula it follows that

$$
\begin{gathered}
\operatorname{str}\left(K^{A} E_{n, m} E_{k, l}\right)=0 \quad \text { if } n \neq l \text { or } m \neq k, \\
\operatorname{str}\left(K^{A} E_{n, m} E_{m, n}\right)=(-1)^{n} n ! m ! \beta^{A 0}(n+m) .
\end{gathered}
$$

In this paper we only consider the even subalgebra of $A q(2 ; 2 \nu)$ spanned by even power polynomials in $a^{ \pm}$which in its turn decomposes into a direct sum of two subalgebras $A q^{ \pm}(2 ; 2 \nu)$ projected out by the projectors

$$
P^{ \pm}=\frac{1}{2}(1 \pm K)
$$

The corresponding bilinear forms read

$$
\operatorname{str}\left(P^{ \pm} E_{n, m} E_{m, n}\right)=(-1)^{n} n ! m ! \beta^{ \pm}(n+m),
$$

where

$$
\beta^{ \pm}(n)=2^{-(n+1)}\left[1 \mp \frac{1}{2}\left(1+(-1)^{n}\right) \frac{2 \nu}{n+1}\right] \prod_{p=0}^{[(n-1) / 2]}\left(1-\frac{4 \nu^{2}}{(2 p+1)^{2}}\right)
$$


For the case of even $n$ under investigation this formula can be equivalently rewritten as

$$
\beta^{ \pm}(2 m)=2^{-(2 m+1)} \prod_{p=-m}^{p=m}\left(1 \mp \frac{2 \nu}{2 p+1}\right) .
$$

From this formula it follows that the bilinear form $\operatorname{str}(A B)$ degenerates for any value of $2 \nu=2 l+1$, where $l$ is an arbitrary integer. As a result, all $P^{+} E_{n, m}$ with $n+m \geq 2 l$ and $P^{-} E_{n, m}$ with $n+m \geq 2(l+1)$ decouple from everything under the supertrace operation. This means that all these higher-order polynomials in $a^{ \pm}$form an ideal $I_{l}^{ \pm}$of $A q^{ \pm}(2 l+1)$ which can be factored out (and in fact factors out automatically under the supertrace operation). As a result one is left with the finite-dimensional factor algebra of lower-order polynomials. Its dimensionality is $l^{2}$ and $(l+1)^{2}$ in the case of $A q^{+}(2 l+1)$ and $A q^{-}(2 l+1)$, respectively [10]. Since this finite-dimensional algebra possesses a non-degenerate bilinear form, one concludes (see e.g. [24]) that it is isomorphic to the matrix algebras $M_{a t}$ and $M a t_{l+1}$, respectively, i.e.

$$
A q^{+}(2 l+1) / I_{l}^{+}=\text {Mat }_{l} \quad A q^{-}(2 l+1) / I_{l}^{-}=\text {Mat }_{(l+1)} .
$$

Let us note that from the basic definition of the supertrace (2.13) it follows that for $2 \nu=2 l+1$

$$
\operatorname{str}\left(P^{+}\right)=-l, \quad \operatorname{str}\left(P^{-}\right)=l+1,
$$

which agrees with the standard definition of the trace of matrix algebras, $\operatorname{tr}(1)=n$ for $M_{a t}{ }_{n}$. The negative relative sign in the relations (2.24) is due to the invariance of the full original algebra with respect to $\nu \leftrightarrow-\nu, K \leftrightarrow-K$ which is equivalent to $P^{ \pm} \leftrightarrow P^{\mp}$, $l \leftrightarrow-(l+1)$.

Taking into account these results we introduce the notation $a\left(\nu \mp \frac{1}{2}\right)$ for the Lie algebras of commutators originating from $A q^{ \pm}(2 \nu \mp 1)$

In accordance with the standard definition, a linear mapping $\rho$ of an algebra $A$ onto itself such that

$$
\rho(a b)=\rho(b) \rho(a), \quad \forall a, b \in A .
$$

is called an antiautomorphism of $A$. The algebra $A q(2 ; 2 \nu)$ admits [10 the following antiautomorphism $\rho\left(P^{ \pm} E_{n, m}\right)=i^{n+m} P^{ \pm} E_{n, m}$. To make sure that the property (2.25) is true it is enough to check that it is in agreement with the defining relations (2.2) and (2.3). Obviously, any antiautomorphism of $A q(2 ; 2 \nu)$ induces some automorphism of the related $a\left(\nu \mp \frac{1}{2}\right)$ algebras of commutators simply by changing a sign. An automorphism $\tau$ related in this way to $\rho$ therefore is

$$
\tau\left(P^{ \pm} E_{n, m}\right)=-i^{n+m} P^{ \pm} E_{n, m} .
$$

By using the automorphism $\tau$ one can project out subsystems of elements satisfying the condition

$$
\tau(a)=a,
$$

which span a proper subalgebra of the original $a\left(\nu \mp \frac{1}{2}\right)$ Lie algebras.

For the case of $2 \nu=2 l+1$ with $l=2 n$ one finds [10 that the respective truncated subalgebras of $A q^{+}\left(l+\frac{1}{2}\right) / I_{l}^{+}$is $s p(2 n) \sim c_{n}$ and of $A q^{-}\left(l+\frac{1}{2}\right) / I_{l}^{-}$is $o(2 n+1) \sim b_{n}$. For 
$l=2 n+1$ one gets $s p(2 n+2) \sim c_{n+1}$ as a truncation of $A q^{+}\left(l+\frac{1}{2}\right) / I_{l}^{+}$and $o(2 n+1) \sim b_{n}$ as a truncation of $A q^{-}\left(l+\frac{1}{2}\right) / I_{l}^{-}$. Note that it is impossible to extract the $o(2 n)=d_{n}$ algebra in this way.

It is important to note that the whole analysis below turns out to be invariant under the action of the automorphism $\tau$. This means that the case of algebras $a(\sigma)$ considered in the sequel of this paper can be trivially truncated to the $b(\sigma)$ and $c(\sigma)$ - type Toda field theories.

\section{Generalized Toda Dynamics}

The infinite-dimensional Lie algebras $a(\mu)$ have been used as the gauge symmetry algebras in the construction of higher-spin gauge theories in three and two space-time dimensions [12, 14]. The $2 d$ gauge fields of this algebra can be written as

$$
W_{\mu}=\sum_{n=0}^{\infty} \frac{1}{n !} W_{\mu \alpha_{1} \ldots \alpha_{n}}^{A}(z) K^{A} a^{\alpha_{1}} \ldots a^{\alpha_{n}},
$$

where $W_{\mu \alpha_{1} \ldots \alpha_{n}}^{A}(z)$ are the higher-spin gauge fields, $z^{\mu}$ denotes the two-dimensional spacetime coordinates and $\mu= \pm$.

The curvatures have the standard form

$$
R_{\nu \mu}=\partial_{\nu} W_{\mu}-\partial_{\mu} W_{\nu}+\left[W_{\nu}, W_{\mu}\right] .
$$

where $\partial_{ \pm}=\frac{1}{\sqrt{2}}\left(\partial_{0} \pm \partial_{1}\right)$.

Setting the curvature to zero allows us to find vacuum solutions of the higher-spin gauge theory. The simplest vacuum solution which describes the anti-de Sitter vacuum geometry is obtained by constraining $W_{\mu}$ to be of the form

$$
W_{\mu}=h_{\mu}^{+}(z) T^{+}+h_{\mu}^{-}(z) T^{-}+\omega_{\mu}(z) T^{0},
$$

where $h_{\mu}^{ \pm}$are zweibein one-forms and $\omega_{\mu}$ is the Lorentz connection one-form. This ansatz is consistent because the generators $T^{ \pm}$and $T^{0}$ span a proper $s l_{2}$ subalgebra of the full algebra. The components of the $s l_{2}$ curvatures then read

$$
R_{\nu \mu}=R_{\nu \mu}^{+} T^{+}+R_{\nu \mu}^{-} T^{-}+R_{\nu \mu}^{0} T^{0},
$$

with

$$
\begin{gathered}
R_{\nu \mu}^{ \pm}=\partial_{\nu} h_{\mu}^{ \pm} \pm \omega_{\nu} h_{\mu}^{ \pm}-(\nu \leftrightarrow \mu), \\
R_{\nu \mu}^{0}=\partial_{\nu} \omega_{\mu}+2 h_{\nu}^{-} h_{\mu}^{+}-(\nu \leftrightarrow \mu) .
\end{gathered}
$$

By using a conformal gauge

$$
h_{\mu}^{ \pm}=\delta_{\mu}^{ \pm} \exp \left(\varphi^{ \pm}\right),
$$

the zero-curvature conditions $R_{\nu \mu}^{ \pm}=0$ allows us to solve for $\omega_{\nu}$

$$
\omega_{ \pm}= \pm \partial_{ \pm} \varphi^{\mp} .
$$


Inserting this into the equation $R_{\nu \mu}^{0}=0$ we are left with the Liouville equation

$$
\partial_{+} \partial_{-}\left(\varphi^{+}+\varphi^{-}\right)=-2 \exp \left[\left(\varphi^{+}+\varphi^{-}\right)\right] .
$$

Note that the fields $\varphi^{ \pm}$appear only in the combination $\varphi^{+}+\varphi^{-}$since the local Lorentz symmetry still remains unfixed. One can take any convenient gauge condition like, say, $\varphi^{-}=0$.

In order to obtain the generalized Toda system we will follow the general zero-curvature approach [1, 0, 16] and consider the following constraints for the higher-spin gauge fields

$$
W_{\mu}=\delta_{\mu}^{+} H^{+}+\delta_{\mu}^{-} H^{-}+\omega_{\mu}\left(T^{0}\right)
$$

where the index \pm indicates that the element $H^{ \pm}$has grade \pm 1 in the sense that $\left[T^{0}, H^{ \pm}\right]$ $= \pm H^{ \pm}$while $\left[T^{0}, \omega_{\mu}\left(T^{0}\right)\right]=0$. The non-trivial curvatures can then be written as

$$
R_{\nu \mu}=R_{\nu \mu}^{+}+R_{\nu \mu}^{-}+R_{\nu \mu}^{0}
$$

where

$$
\begin{gathered}
R_{\nu \mu}^{ \pm}=\delta_{\mu}^{ \pm} \partial_{\nu} H^{ \pm}-\delta_{\nu}^{ \pm} \partial_{\mu} H^{ \pm}+\delta_{\mu}^{ \pm}\left[\omega_{\nu}, H^{ \pm}\right]-\delta_{\nu}^{ \pm}\left[\omega_{\mu}, H^{ \pm}\right] \\
R_{\nu \mu}^{0}=\partial_{\nu} \omega_{\mu}+\delta_{\nu}^{+} \delta_{\mu}^{-}\left[H^{+}, H^{-}\right]-\nu \leftrightarrow \mu
\end{gathered}
$$

A convenient parametrization of $H^{ \pm}$is

$$
H^{+}=e^{\phi_{+}} T^{+} e^{-\phi_{+}}, \quad H^{-}=e^{-\phi_{-}} T^{-} e^{\phi_{-}},
$$

where $\phi_{ \pm}$are general functions of the generator $T_{0}$ and of $K$.

Imposing the zero-curvature conditions $R_{\nu \mu}^{ \pm}=0$ one finds

$$
\omega_{ \pm}= \pm \partial_{ \pm} \phi_{\mp}
$$

while from $R^{0}=0$ one gets

$$
\partial_{-} \partial_{+}\left(\phi_{+}+\phi_{-}\right)=\left[H^{+}, H^{-}\right] .
$$

Again we have a Lorentz symmetry-type gauge ambiguity which can be used to set one of the $\phi_{ \pm}$fields to zero. From now on we will use the gauge

$$
\phi_{+}=0, \rightarrow \omega_{-}=0, \quad H^{+}=T^{+}
$$

and denote $\phi_{-}=\phi$ using the label \pm in a different context from above to indicate projections by $P^{ \pm}(2.19)$. The general expansion is then

$$
\phi^{ \pm}=P^{ \pm} \phi=\sum_{n=0}^{\infty} P^{ \pm} \phi_{n}^{ \pm} h_{n},
$$

where $h_{n}$ is defined in $(2.10)$.

In the sequel we will show how these equations include the $A_{n}$ Toda equations for the specific values of the parameter $2 \nu=2 n+1$. For other values of $\nu$ the same equations describe an infinite component system. 
Note that by rescaling the $\phi$ fields as $\phi \rightarrow \beta \phi$ one can rewrite the equations (3.16) in the equivalent form

$$
\partial_{-} \partial_{+} \phi=\beta^{-1}\left[T^{+}, e^{-\beta \phi} T^{-} e^{\beta \phi}\right]
$$

Since in this paper we only treat the classical case we will use this freedom to set $\beta$ to one.

When the bilinear form (2.15) is non-degenerate, i.e. for all $2 \nu \neq 2 l+1$, where $l$ is an integer, the equations of motion (3.16) can be elevated to an action

$$
S^{ \pm}=-\int d^{2} z \operatorname{str}\left(P^{ \pm}\left(\phi \partial_{-} \partial_{+} \phi-2 H^{+} H^{-}\right)\right) .
$$

When $2 \nu=2 l+1$, the null-vector ideals do not contribute to the supertrace. As a result the action reduces to the finite-dimensional system automatically.

The action (3.20) is conformally invariant under transformations of the form

$$
\delta \phi=u\left(z^{+}\right) \partial_{+} \phi\left(T^{0}, z\right)+\partial_{+} u\left(z^{+}\right) T^{0},
$$

where $u\left(z^{+}\right)$is an arbitrary parameter function. We will come back to the analysis of the symmetries of the action (3.20) in section 5 .

\section{Comparison to Toda Field Theories}

In order to make contact for the action (3.20) with the usual Toda systems one first of all must be able to compute expressions of the form

$$
V^{ \pm}(\nu ; \phi)=-2 \operatorname{str}\left(P^{ \pm} T^{+} e^{-\phi} T^{-} e^{\phi}\right)
$$

for any $\phi$ of the form (3.18). This can be done in two steps. First one moves $T^{-}$through the exponential reducing the problem to compute expressions of the form

$$
Z^{ \pm}(\nu ; \alpha)=\operatorname{str}\left(P^{ \pm} \exp \sum_{n}\left(\alpha_{n} h_{n}\right)\right) .
$$

The first step can be carried out by virtue of the following useful formula

$$
a^{-} h_{n}=\tilde{h}_{n} a^{-}
$$

where

$$
\tilde{h}_{n}=\sum_{l=0}^{n} A_{l}^{n}(\nu) h_{n-l}
$$

and

$$
A_{l}^{n}(\nu)=\frac{1}{2^{l-1}} \frac{n !}{(n-l) !} \prod_{m=1}^{l-1}\left(1-\frac{2 \nu K}{2 n+1-2 m}\right) ; \quad A_{0}^{n}(\nu)=1, \quad A_{1}^{n}(\nu)=n .
$$

The formula (4.3) can be derived by using properties of Weyl ordered polynomials of $a^{ \pm}$. By using this formula twice one arrives at a similar expression for the case when $T^{-}$ is commuted through $h_{n}$

$$
T^{-} h_{n}=\tilde{\tilde{h}}_{n} T^{-}
$$


With the aid of this formula one obtains

$$
\begin{aligned}
\operatorname{str}\left(P^{ \pm} T^{+} e^{-\phi} T^{-} e^{\phi}\right) & =\operatorname{str}\left(P^{ \pm} T^{-} T^{+} e^{(\tilde{\tilde{\phi}}-\phi)}\right) \\
& =\frac{1}{4} \operatorname{str}\left(P^{ \pm}\left(\left(h_{1}\right)^{2}+2 h_{1}+\frac{1}{4}\left(3 \pm 4 \nu-4 \nu^{2}\right)\right) e^{(\tilde{\tilde{\phi}}-\phi)}\right),
\end{aligned}
$$

where we have made use of the formula (2.6).

As a result the potential (4.1) can be computed from the generating function $Z^{ \pm}(\nu ; \alpha)$ (4.2) in the following way

$$
V^{ \pm}(\nu ; \phi)=-\left.\frac{1}{2}\left(\frac{\partial^{2}}{\partial \alpha_{1}^{2}}+2 \frac{\partial}{\partial \alpha_{1}}+\frac{1}{4}\left(3 \pm 4 \nu-4 \nu^{2}\right)\right) Z^{ \pm}(\nu ; \alpha)\right|_{\alpha_{n}=\tilde{\tilde{\phi}}_{n}(z)-\phi_{n}(z)} .
$$

Let us now explain how one can compute generating functions of this form. First, one computes the simplest generating function

$$
Y^{ \pm}(\nu ; \alpha)=\operatorname{str}\left(P^{ \pm} \exp \left(\alpha h_{1}\right)\right)
$$

which can be shown to be

$$
Y^{ \pm}(\nu ; \alpha)=\frac{1}{2}\left(\frac{\operatorname{ch}(\nu \alpha)}{\operatorname{ch}\left(\frac{\alpha}{2}\right)} \mp \frac{\operatorname{sh}(\nu \alpha)}{\operatorname{sh}\left(\frac{\alpha}{2}\right)}\right)=\mp \frac{\operatorname{sh}\left(\left(\nu \mp \frac{1}{2}\right) \alpha\right)}{\operatorname{sh}(\alpha)} .
$$

In order to derive this formula one starts by differentiating $Y(\alpha)$ with respect to $\alpha$. This brings down $h_{1}=\frac{1}{2}\left\{a^{-}, a^{+}\right\}$. By using the properties of the supertrace operation (2.12) and the simplest version of the formula (4.3)

$$
a^{-} h_{1}=\left(h_{1}+1\right) a^{-}
$$

one gets

$$
\begin{aligned}
& \frac{\partial}{\partial \alpha} Y^{ \pm}(\nu ; \alpha)=\frac{1}{2} \operatorname{str}\left(P^{ \pm} a^{-} a^{+} e^{\alpha h_{1}}\right)-\frac{1}{2} \operatorname{str}\left(P^{\mp} a^{-} a^{+} e^{\alpha\left(h_{1}+1\right)}\right) \\
& =\frac{1}{2} \operatorname{str}\left(P^{ \pm}\left(\frac{\partial}{\partial \alpha}+\frac{1}{2}(1 \pm 2 \nu)\right) e^{\alpha h_{1}}\right)-\frac{1}{2} e^{\alpha} \operatorname{str}\left(P^{\mp}\left(\frac{\partial}{\partial \alpha}+\frac{1}{2}(1 \mp 2 \nu)\right) e^{\alpha h_{1}}\right)
\end{aligned}
$$

From this we read off the following differential equations with respect to $\alpha$

$$
\frac{\partial}{\partial \alpha} Y^{ \pm}+e^{\alpha} \frac{\partial}{\partial \alpha} Y^{\mp}=\frac{1}{2}(1 \pm 2 \nu) Y^{ \pm}-\frac{1}{2} e^{\alpha}(1 \mp 2 \nu) Y^{\mp}
$$

The initial data which follow from (2.13) are

$$
Y^{ \pm}(\nu ; 0)=\frac{1}{2}(1 \mp 2 \nu) .
$$

The formula (4.10) is then a unique solution of equation (4.13) under the boundary conditions $(4.14)$. 
Let us now consider the general generating function (4.2). It follows that

$$
\frac{\partial Z^{ \pm}(\nu ; \alpha)}{\partial \alpha_{n+1}}=\operatorname{str}\left(P^{ \pm} h_{n+1} \exp \left(\sum_{n} \alpha_{n} h_{n}\right)\right) .
$$

One can prove the following relation

$$
P^{ \pm} h_{n+1}=P^{ \pm} h_{n} h_{1}+P^{ \pm} d_{ \pm}^{n}(\nu) h_{n-1}
$$

where

$$
d_{ \pm}^{n}(\nu)=\frac{1}{4} n^{2}\left(1 \mp \frac{2 \nu}{2 n+1}\right)\left(1 \pm \frac{2 \nu}{2 n-1}\right)
$$

The equations (4.16) and (4.17) are derived by using the properties of Weyl ordered polynomials in $a^{ \pm}$as well as the commutation relations (2.2).

It then follows that the generating function (4.2) satisfies the following set of differential equations

$$
\left(\frac{\partial}{\partial \alpha_{n+1}}-\frac{\partial}{\partial \alpha_{1}} \frac{\partial}{\partial \alpha_{n}}-d_{ \pm}^{n}(\nu) \frac{\partial}{\partial \alpha_{n-1}}\right) Z^{ \pm}(\nu ; \alpha)=0 .
$$

This equation can then be used in an iterative way to solve for $Z^{ \pm}(\nu ; \alpha)$ once one has solved for the $Z^{ \pm}\left(\nu ; \alpha_{0}, \alpha_{1}, 0,0 \ldots\right)=e^{\alpha_{0}} Y^{ \pm}\left(\nu, \alpha_{1}\right)$ using $Z^{ \pm}\left(\nu ; \alpha_{0}, \alpha_{1}, \ldots, \alpha_{n}, 0, \ldots\right)$ successively as initial data. For example, when the initial data are of the form

$$
Z^{ \pm}\left(\nu ; \alpha_{0}, \alpha_{1}, 0,0 \ldots\right)=e^{\alpha_{0}} \sum_{k} c_{k} e^{u_{k}^{1} \alpha_{1}}
$$

with some coefficients $c_{k}$ and $u_{k}^{1}$, then the solution of (4.18) reads

$$
Z^{ \pm}(\nu ; \alpha)=\sum_{k} c_{k} \exp \left(\alpha_{0}+\sum_{l} u_{k}^{l} \alpha_{l}\right)
$$

where the coefficients $u_{k}^{l}$ are determined from the equation

$$
u_{k}^{n+1}-u_{k}^{n} u_{k}^{1}-d_{ \pm}^{n}(\nu) u_{k}^{n-1}=0 .
$$

This concludes the general scheme of expressing the potential term in the action in terms of the component fields $\phi_{n}$.

Using (2.20) and (2.22) one can easily find the kinetic part of the action

$$
S_{k i n}^{ \pm}=-\int d^{2} z \operatorname{str}\left(P^{ \pm} \phi \partial_{-} \partial_{+} \phi\right)=-\sum_{n=0}^{\infty} b_{n}^{ \pm} \int d^{2} z \phi_{n}^{ \pm} \partial_{-} \partial_{+} \phi_{n}^{ \pm},
$$

where

$$
b_{n}^{ \pm}=(-1)^{n} 2^{-(2 n+1)}(n !)^{2} \prod_{l=-n}^{n}\left(1 \mp \frac{2 \nu}{2 l+1}\right) .
$$

Let us now check these general formulae for the lowest finite systems. For the particular cases of $2 \nu=2 l+1$, where $l$ is an integer, the computation is simplified since only a finite number of fields contribute because all terms involving $P^{ \pm} h_{n}$ with $n \geq l+\frac{1}{2} \mp \frac{1}{2}$ decouple from the supertrace. As a particular manifestation of this phenomenon the coefficients 
(4.23) all vanish starting from $n=l+\frac{1}{2} \mp \frac{1}{2}$. For $2 \nu \neq 2 l+1$ all $b_{n}^{ \pm}$are non-vanishing. In this case however the coefficients with $n$ high enough take both positive and negative values so that the full infinite system involves ghosts. Due to the invariance of the full original algebra with respect to $\nu \leftrightarrow-\nu, K \leftrightarrow-K$ which for $\nu=2 l+1$ is equivalent to $P^{ \pm} \leftrightarrow P^{\mp}, l \leftrightarrow-(l+1)$ we only need to consider the case of non-negative $\nu$.

According to 4.8$)$ one needs to know the generating function $Z^{ \pm}(\nu ; \alpha)$ and the difference

$$
\hat{\phi}(z)=\tilde{\tilde{\phi}}(z)-\phi(z) \text {. }
$$

Let us first note that the components $\phi_{0}^{ \pm}$do not contribute to the potential since $\tilde{\tilde{\phi}}_{0}^{ \pm}=\phi_{0}^{ \pm}$, which is in fact obvious from (4.1). This is a particular manifestation of the general property that if $\phi$ is such that $\phi_{n}^{ \pm}=0$ for $n \geq n_{0}$ then $\hat{\phi}_{n}^{ \pm}=0$ for $n \geq n_{0}-1$.

For $l=0$ one easily finds that the potential 4.1$) V^{ \pm}(1 ; \phi)=0$ since the only nonvanishing component of $\hat{\phi}$ is $\hat{\phi}_{0}(z)$ and according to 4.10$) Y_{1}^{ \pm}(0 ; \alpha)=\frac{1}{2}(1 \mp 1)$ so that inserting it back into (4.8) one finds that the terms involving derivatives trivialize while the constant factor turns out to be proportional to $(1 \pm 1)$ and therefore the potential vanishes identically.

Let us so consider the case $l=1$, i.e. $2 \nu=3$. For the " + " case one concludes from the formula (2.23) that again the factor algebra is one-dimensional ( $A_{0}$ "), i.e. only the constant mode contributes under the supertrace leading to a vanishing potential. Hence we now discuss the " - " case of $A_{1}$ where the $h_{1}$ - dependent terms have to be taken into account. From (4.2), (4.9) and (4.10) it follows that

$$
Z^{-}(3 / 2 ; \alpha)=e^{\alpha_{0}} Y^{-}\left(3 / 2 ; \alpha_{1}\right)
$$

with

$$
Y^{-}(3 / 2 ; \alpha)=2 \operatorname{ch}(\alpha) \text {. }
$$

Inserting all this into (4.8) and taking into account that $\hat{\phi}_{0}^{-}=2 \phi_{1}^{-}$and $\hat{\phi}_{1}^{-}=0$ one finds

$$
V^{-}\left(3 / 2 ; \phi^{-}\right)=2 \exp \left(2 \phi_{1}^{-}\right)
$$

With the proper rescaling of the $\phi_{1}$ field we recognize the Liouville potential.

Next we treat the case $l=2$, i.e. $2 \nu=5$. The calculation in the " + " case of $A_{1}$ follows exactly the lines of the " - " case for $l=1$. Also in this case all $h_{n}$ with $n>1$ decouple. The final result for the potential only differs in the overall sign. In the " - " case also the $h_{2}$ terms in $\phi^{-}$couple in the supertrace. From (4.6) one easily finds for arbitrary $\nu$

$$
T^{-} h_{2}=\left(h_{2}+4 h_{1}+4\right) T^{-} .
$$

From this equation and (4.11) it follows that

$$
\hat{\phi}^{-}=4 \phi_{2}^{-} h_{1}+4 \phi_{2}^{-}+2 \phi_{1}^{-} .
$$

The function $Y^{ \pm}(\alpha)$ has the following form

$$
Y^{-}(5 / 2 ; \alpha)=\frac{\operatorname{sh}(3 \alpha)}{\operatorname{sh}(\alpha)}=e^{2 \alpha}+1+e^{-2 \alpha} \text {. }
$$


From (4.8) it now follows that

$$
V^{-}(5 / 2 ; \phi)=4 e^{2 \phi_{1}^{-}}\left(e^{4 \phi_{2}^{-}}+e^{-4 \phi_{2}^{-}}\right) .
$$

Finally we investigate the case $l=3$. The " + " case of $A_{2}$ is again parallel to the " - " case for $l=2$. As in that case $h_{n}$ with $n>2$ decouple and the final expression for the potential only differs from (4.31) by a minus sign. In the " - " case also the $h_{3}$ terms couple.

The first step is to derive $\hat{\phi}^{-}$. From (4.6) we find

$$
T^{-} h_{3}=\left(h_{3}+6 h_{2}+12 h_{1}+9+\frac{4 \nu^{2}}{5}-\frac{4 \nu K}{5}\right) T^{-} .
$$

Using also (4.28) and (4.3) one finds for $2 \nu=7$ and $K=-1$

$$
\hat{\phi^{-}}=6 \phi_{3}^{-} h_{2}+\left(12 \phi_{3}^{-}+4 \phi_{2}^{-}\right) h_{1}+\frac{108}{5} \phi_{3}^{-}+4 \phi_{2}^{-}+2 \phi_{1}^{-} .
$$

The next step is to compute the generating function $Z^{-}(7 / 2 ; \alpha)$. In order to do that we first rewrite $Y^{-}(7 / 2 ; \alpha)$ as

$$
Y^{-}(7 / 2 ; \alpha)=e^{3 \alpha}+e^{\alpha}+e^{-\alpha}+e^{-3 \alpha}
$$

so that

$$
Z^{-}\left(7 / 2 ; \alpha_{0}, \alpha_{1}, 0,0, \ldots\right)=e^{\alpha_{0}}\left(e^{3 \alpha_{1}}+e^{\alpha_{1}}+e^{-\alpha_{1}}+e^{-3 \alpha_{1}}\right) .
$$

Now it is easy to integrate the equation (4.18) at $n=1$ to obtain the full generating function $Z^{-}(7 / 2 ; \alpha)$

$$
Z^{-}(7 / 2 ; \alpha)=e^{\alpha_{0}}\left(e^{4 \alpha_{2}+3 \alpha_{1}}+e^{-4 \alpha_{2}+\alpha_{1}}+e^{-4 \alpha_{2}-\alpha_{1}}+e^{4 \alpha_{2}-3 \alpha_{1}}\right) .
$$

Inserting this generating functional with $\hat{\phi}^{-}$from (4.33) into (4.8) gives the potential

$$
\begin{aligned}
V^{-}(7 / 2 ; \phi) \quad & =2\left(3 \exp \left(\frac{48}{5} \phi_{3}^{-}+8 \phi_{2}^{-}+2 \phi_{1}^{-}\right)\right. \\
& \left.+4 \exp \left(-\frac{72}{5} \phi_{3}^{-}+2 \phi_{1}^{-}\right)+3 \exp \left(\frac{48}{5} \phi_{3}^{-}-8 \phi_{2}^{-}+2 \phi_{1}^{-}\right)\right) .
\end{aligned}
$$

In principle one can derive in this way the potential for any finite system. The feature that the potential for the " + " case with $2 \nu=2 l+1$ coincides with the negative of the potential for the " -" case with $2 \nu=2 l-1$ is a general feature which follows from (2.23) and (2.24). In the sequel we only consider the " - " cases.

We then compare the various cases to the standard Toda actions

$$
S_{l}=-\int d^{2} z\left(\sum_{a, b=1}^{l} K^{-1 a b} q_{a} \partial_{-} \partial_{+} q_{b}+\sum_{a=1}^{l} e^{-q_{a}}\right),
$$

where $K_{a b}$ is the $A_{l}$ Cartan matrix, i.e. the non-vanishing components of $K_{a b}$ are $K_{a a}=2$, $K_{a, a-1}=K_{a-1, a}=-1$. 
For the Liouville case which corresponds to $l=1$ the action can always be reduced to the form (4.38).

For the case $l=2$ we find from (4.31) that the relevant field redefinitions are

$$
q_{1}=-2 \phi_{1}^{-}-4 \phi_{2}^{-}-\ln 4, \quad q_{2}=-2 \phi_{1}^{-}+4 \phi_{2}^{-}-\ln 4 .
$$

With this substitution one arrives at the formula (4.38).

For the case $l=3$ we proceed in the same manner and find from (4.37) that the relevant field redefinitions are

$$
\begin{aligned}
& q_{1}=-\left(\frac{48}{5} \phi_{3}^{-}+8 \phi_{2}^{-}+2 \phi_{1}^{-}+\ln 6\right) \\
& q_{2}=-\left(-\frac{72}{5} \phi_{3}^{-}+2 \phi_{1}^{-}+\ln 8\right) \\
& q_{3}=-\left(\frac{48}{5} \phi_{3}^{-}-8 \phi_{2}^{-}+2 \phi_{1}^{-}+\ln 6\right) .
\end{aligned}
$$

By substituting these field variables into the action we again find the canonical form (4.38).

Let us also mention that one can consider the one-dimensional Toda systems within this formalism too. The equation of motion then reads

$$
\ddot{\phi}=\left[H^{+}, H^{-}\right] \text {. }
$$

By introducing the canonical momentum $\pi$ one arrives at the Hamiltonian equations

$$
\dot{\phi}=\pi, \quad \dot{\pi}=\left[H^{+}, H^{-}\right]
$$

and it is easy to formulate it in a Lax pair form

$$
\begin{gathered}
L=\pi I+\frac{1}{\sqrt{2}} K\left(H^{+}+H^{-}\right), \\
A=\frac{1}{\sqrt{2}} K\left(H^{+}-H^{-}\right), \\
\dot{L}=[A, L] .
\end{gathered}
$$

\section{$5 \quad$ Higher-spin symmetries}

The generalized Toda field equations were derived in (3.16) from the condition that the higher-spin curvatures (3.11) be zero. It is then natural to seek the symmetries of the Toda equations as specific higher spin gauge transformations

$$
\delta W_{\mu}=\partial_{\mu} \epsilon+\left[W_{\mu}, \epsilon\right]
$$

For example the conformal transformations of the $\phi$ - field (3.21) can be derived from the gauge transformations as follows. Let us consider gauge parameters of the form

$$
\epsilon=\epsilon^{+}+\epsilon^{0}
$$


with

$$
\epsilon^{+}=u(z) T^{+}, \quad \epsilon^{0}=v(z) T^{0} .
$$

One can then ask which gauge transformations leave invariant the Toda ansatz (3.10) and (3.17) for gauge fields. Note that the gauge condition (3.17) is a convenient one because it implies that one has to require

$$
\delta W_{\mu}^{\text {positive }}=0,
$$

where $W_{\mu}^{\text {positive }}$ denotes all gauge fields with positive gradings with respect to $T^{0}$. (The gauge condition (3.17) tells us that the field $\mathrm{H}^{+}$is constant and therefore its variation vanishes.) When analyzing the anti-conformal invariances it is more convenient to fix another gauge $\phi_{-}=0$.

The only non-trivial condition for the simplest case under consideration is

$$
0=\delta W_{\mu}^{+}=\partial_{\mu} \epsilon^{+}+\left[W_{\mu}^{+}, \epsilon^{0}\right]+\left[W_{\mu}^{0}, \epsilon^{+}\right],
$$

where $W_{\mu}^{+}=\delta_{\mu}^{+} H^{+}$and $W_{\mu}^{0}=\omega_{\mu}\left(T^{0}\right)$. Now setting $\mu=-$ and taking into account that $W_{-}^{0}=0$ due to the gauge condition (3.17) one finds

$$
\partial_{-} \epsilon^{+}=0 \text {. }
$$

For $\mu=+$ one finds taking into account that $H^{+}=T^{+}$due to (3.14) and the gauge condition (3.17):

$$
0=\partial_{+} \epsilon^{+}+\left[T^{+}, \epsilon^{0}\right]+\left[\partial_{+} \phi, \epsilon^{+}\right] .
$$

One can solve this for $\epsilon^{0}$ with the result

$$
\epsilon^{0}=\partial_{+} \phi u\left(z^{+}\right)+T_{0} \partial_{+} u\left(z^{+}\right)
$$

that gives exactly the conformal transformation (3.21) for $\phi$, taking into account that

$$
\delta H^{-}=\left[H^{-}, \epsilon^{0}\right]
$$

because there are no negative terms in $\epsilon$.

The above analysis generalizes to higher-spin conformal transformations as follows. The decomposition (5.2) relevant for the conformal symmetry generalizes to

$$
\epsilon^{s}=\sum_{n=0}^{s-1} \epsilon^{s, n},
$$

where

$$
\left[T^{0}, \epsilon^{s, n}\right]=n \epsilon^{s, n}
$$

and $s$ is the spin of the corresponding higher-spin current so that $s=2$ for the case of conformal symmetry.

As before we fix the gauge (3.17) and impose the conditions (5.4). This leads to the following chain of equations generalizing (5.5):

$$
0=\delta W_{\mu}^{n}=\partial_{\mu} \epsilon^{s, n}+\left[W_{\mu}^{+}, \epsilon^{s, n-1}\right]+\left[W_{\mu}^{-}, \epsilon^{s, n+1}\right]+\left[W_{\mu}^{0}, \epsilon^{s, n}\right]
$$


for $n>0, \mu=+$ and $n \geq 0, \mu=-$. As a result one finds, respectively

$$
0=\delta W_{+}^{n}=\partial_{+} \epsilon^{s, n}+\left[T^{+}, \epsilon^{s, n-1}\right]+\left[\partial_{+} \phi, \epsilon^{s, n}\right] ; \quad n>0
$$

and

$$
0=\delta W_{-}^{n}=\partial_{-} \epsilon^{s, n}+\left[H^{-}, \epsilon^{s, n+1}\right] ; \quad n \geq 0
$$

taking into account (3.15) for the particular gauge (3.17).

One observes that for the terms with highest grading in the expansion (5.10) the consistency of the above equations requires, respectively

$$
\left[T^{+}, \epsilon^{s, s-1}\right]=0
$$

and

$$
\partial_{-} \epsilon^{s, s-1}=0
$$

i.e. the top parameters $\epsilon^{s, s-1}$ must be $z^{-}$- independent highest weight vectors of the basic $s l_{2}$ algebra. $\epsilon^{s, s-1}\left(z^{+}\right)$serve as independent parameters of the higher-spin symmetries.

One can then use the basic relations (5.13) to reconstruct all lower $\epsilon^{s, n}$ with $0<n<$ $s-1$ in terms of $\epsilon^{s, s-1}$ and $\left(\partial_{+}-\right.$derivatives of $) \phi$,

$$
\epsilon^{s, p}=-t\left(\mathcal{D} \epsilon^{s, p+1}\right)+\epsilon_{+}^{s, p},
$$

where $\epsilon_{+}^{s, p}$ are some quantities obeying the condition

$$
\left[T^{+}, \epsilon_{+}^{s, p}\right]=0
$$

(i.e. highest weight vectors of $s l_{2}$ ), $\mathcal{D}$ is the Lorentz covariant derivative

$$
\mathcal{D} A=\partial_{+} A+\left[\partial_{+} \phi, A\right]
$$

and $t$ is defined in such a way that

$$
\left[T^{+}, t(A)\right]=A
$$

for any operator $A \in A(2 ; 2 \nu)$ which has a non-negative grading (and not a constant in the Weyl ordering).

In what follows we use the following definition for $t$ in the basis of Weyl ordered polynomials used in (2.9)

$$
t\left(E_{n, m}\right)=-\frac{1}{m+1} E_{n-1, m+1} \quad n>0 ; \quad t\left(E_{0, m}\right)=0 .
$$

An important property of this operation is that it is skewsymmetric in the following sense

$$
\operatorname{str}(A t(B))=-\operatorname{str}(t(A) B) .
$$

It is easy to see that such a definition of $t$ leads to the following relations

$$
\left[T^{+}, t(x)\right]=x-\Pi_{-}(x), \quad t\left(\left[T^{+}, x\right]\right)=x-\Pi_{+}(x),
$$


where $\Pi_{+}$and $\Pi_{-}$are respectively projection operators to highest weight and lowest weight components, i.e.

$$
\begin{array}{lll}
\Pi_{-}\left(E_{0, m}\right)=E_{0, m} ; & \Pi_{-}\left(E_{n, m}\right)=0 & n>0, \\
\Pi_{+}\left(E_{n, 0}\right)=E_{n, 0} ; & \Pi_{+}\left(E_{n, m}\right)=0 & m>0 .
\end{array}
$$

Also in what follows we will use the following projection operator on the neutral subspace

$$
\Pi_{0}\left(E_{n, n}\right)=E_{n, n} \equiv h_{n} ; \quad \Pi_{0}\left(E_{n, m}\right)=0 \quad n \neq m .
$$

The iterative solution of equation (5.17) (equivalently (5.13)) for $\epsilon^{s, 0}$ can be written in the following form

$$
\epsilon^{s, 0}=\sum_{p=0}^{s-1}(-t \mathcal{D})^{p} \epsilon_{+}^{s, p},
$$

where $\epsilon_{+}^{s, s-1}=\epsilon^{s, s-1}$.

In order to guarantee that $(5.14)$ is also true it suffices to ensure that

$$
\Pi_{+}\left(\partial_{-} \epsilon^{s, p}+\left[H^{-}, \epsilon^{s, p}\right]\right)=0
$$

and take into account the Toda equations. Indeed, using the fact that the equations of motion result from the zero-curvature conditions one observes that

$$
\left[\partial_{-}+H^{-},\left[\mathcal{D}+T^{+}, \epsilon\right]\right]-\left[\mathcal{D}+T^{+},\left[\partial_{-}+H^{-}, \epsilon\right]\right]=0
$$

when (3.19) is true. Taking into account (5.13) one finds that

$$
\left[T^{+},\left(\partial_{-}(\epsilon)+\left[H^{-}, \epsilon\right]\right)\right]=-\mathcal{D}\left(\partial_{-}(\epsilon)+\left[H^{-}, \epsilon\right]\right) .
$$

From here it follows that the components of $\left(\partial_{-}(\epsilon)+\left[H^{-}, \epsilon\right]\right)$ with all possible gradings vanish provided that the highest weight components do (that allows one to start the inductive proof with the vanishing right-hand side of (5.30) with the highest possible grading). This is indeed guaranteed by the condition (5.28).

In order to satisfy (5.28) one has to fix the free parameters $\epsilon_{+}^{s, p}$ in (5.27) in an appropriate way. In the next section we will give a constructive method which will fix these parameters.

Since $\epsilon$ in (5.10) is supposed to involve only non-negative gradings, one finds from the transformation law of $H^{-}$that

$$
\delta H^{-}=\left[H^{-}, \epsilon^{s, 0}\right]
$$

and therefore for a spin $s$ higher-spin transformation

$$
\delta \phi=\epsilon^{s, 0}
$$

i.e. the parameters $\epsilon^{s, 0}$ serve as spin $s$ variations of the physical fields.

So far we have studied the invariance at the level of the equations of motion. Let us investigate whether this invariance also works for the action (3.20). It will turn out that 
the potential and kinetic terms in the action (3.20) are invariant separately. Indeed, the variation of the potential term is

$$
\delta^{p o t} S^{ \pm}=2 \operatorname{str} \int d^{2} z\left(P^{ \pm} H^{+}\left[H^{-}, \epsilon^{s, 0}\right]\right)
$$

where we have taken into account that in the gauge under consideration $H^{+}=T^{+}$and the variation of $H^{-}$is given in (5.31). With the aid of the basic property of supertrace (for even higher spin parameters) one transforms (5.33) to

$$
\delta^{p o t} S^{ \pm}=-2 \operatorname{str} \int d^{2} z\left(P^{ \pm}\left[H^{+}, \epsilon^{s, 0}\right] H^{-}\right)
$$

and then by virtue of the basic relations (5.13) to

$$
\delta^{p o t} S^{ \pm}=2 \operatorname{str} \int d^{2} z\left(P^{ \pm} \mathcal{D}\left(\epsilon^{s, 1}\right) H^{-}\right)=-2 \operatorname{str} \int d^{2} z\left(P^{ \pm} \epsilon^{s, 1} \mathcal{D}\left(H^{-}\right)\right)
$$

(recall that $\mathcal{D}\left(H^{-}\right)=R_{+-}^{-}=0$ is the defining relation for the Lorentz connection $\omega_{+}$).

Now let us consider the kinetic term. According to (3.20)

$$
\delta S_{\text {kin }}^{ \pm}=-2 \int d^{2} z \operatorname{str}\left(P^{ \pm} \epsilon^{s, 0} \partial_{+} \partial_{-} \phi\right)
$$

This variation would reduce to an integral of a full $\partial_{-}$derivative if there exist such higherspin currents $J^{s}\left(z^{-}\right)$, the functionals of $\phi\left(z^{+}, z^{-}\right)$at fixed $z_{-}$and of higher spin symmetry parameters, that their variation with respect to the dynamical fields $\phi$ has the form

$$
\delta J^{s \pm}\left(z^{-}\right)=\int d z_{+} \operatorname{str}\left(P^{ \pm} \epsilon^{s, 0} \partial_{+} \delta \phi\right)
$$

Indeed, in this case the variation of the kinetic term amounts to the total derivative

$$
\delta^{k i n} S^{ \pm}=-2 \int d z^{-} \partial_{-} J^{s \pm}
$$

The higher-spin currents (5.37) indeed exist analogously to the case of the ordinary Toda lattice [2]- [6], [17, 18]. The relevant language here is that of Hamiltonian dynamics. Namely, we identify $z^{-}$with the time coordinate while $z^{+}$is to be regarded as a spatial one. Later on we will assume that all relations are valid for equal time variable $z^{-}$which is not written down explicitly in most cases. Also, we will sometimes use $z$ instead of $z^{+}$. In accordance with (3.20) the explicit form of the Hamiltonian is

$$
\mathcal{H}^{ \pm}=-2 \int d z \operatorname{str}\left(P^{ \pm} H^{+} H^{-}\right)
$$

Let us introduce the notation

$$
\langle\xi, \phi\rangle=\int d z \operatorname{str} \xi(z) \phi(z)
$$

where $\xi(z)$ is an arbitrary grade zero element of the algebra $A q(2 ; 2 \nu)$ regarded as a field independent parameter and having sufficiently smooth behavior at infinity in $z$ so that one 
can freely integrate by parts any terms involving $\xi$. Note that according to the defining property of the supertrace (2.12) one has for the integer spin case under consideration that

$$
\langle\xi, \eta\rangle=\langle\eta, \xi\rangle
$$

Also let us use the notation

$$
\phi^{\prime}=\partial_{+} \phi
$$

So that, according to $(5.40)$

$$
\left\langle\xi^{\prime}, \eta\right\rangle+\left\langle\xi, \eta^{\prime}\right\rangle=0
$$

Next we define Poisson brackets $\{$,$\} for the dynamical variables \phi$ as

$$
\left\{\langle\xi, \phi\rangle,\left\langle\eta, \phi^{\prime}\right\rangle\right\}=\langle\xi, \eta\rangle
$$

for field independent $\xi$ and $\eta$.

Since the right hand side of (5.44) is field independent the Jacobi identities are trivially satisfied. It is worth mentioning that when the bilinear form (5.40) is nondegenerate, the definition (5.44) induces a Poisson bracket structure on the dynamical variables $\phi$ themselves. In particular, for this case

$$
\left\{\langle\xi, \phi\rangle, \phi^{\prime}\right\}=\xi
$$

When the bilinear form (5.40) degenerates, the relation (5.44) induces a Poisson bracket structure on the factor algebra over null vectors of the bilinear form (5.40). This is enough because the ideals formed by null vectors of the bilinear form (5.40) do not contribute to any expression involving the supertrace and in particular to the action (3.20). In other words one can always use (5.45) inside the supertrace operation.

Our aim is to prove that there exist such currents that

$$
\left\{\langle\xi, \phi\rangle, J^{s}\right\}=\left\langle\xi, \epsilon^{s, 0}\right\rangle
$$

since this condition is equivalent to the condition (5.37), i.e. the vector fields $\epsilon^{s, 0}$, which act in the space of the field variables $\phi$, are Hamiltonian. In the sequel we will not explicitly indicate the \pm projections by $P^{ \pm}$. All calculations will be insensitive to these projectors and they can always be introduced in the final formulae.

The consistency conditions for this equation require

$$
\left\{\langle\eta, \phi\rangle,\left\langle\xi, \epsilon^{s, 0}\right\rangle\right\}=\left\{\langle\xi, \phi\rangle,\left\langle\eta, \epsilon^{s, 0}\right\rangle\right\}
$$

Provided that the consistency conditions are satisfied, one can derive the following explicit formula for $J^{s}$ in terms of $\epsilon^{s, 0}$

$$
J^{s}=\int_{0}^{1} d \tau\left\langle\phi^{\prime}, \epsilon^{s, 0}(\tau \phi)\right\rangle .
$$

Indeed, inserting this into (5.46) and using (5.47) one easily shows that the left hand side of (5.46) amounts to

$$
\int_{0}^{1} d \tau \frac{\partial}{\partial \tau}\left(\tau\left\langle\xi, \epsilon^{s, 0}(\tau \phi)\right\rangle\right)=\left\langle\xi, \epsilon^{s, 0}\right\rangle
$$


In the next section we will describe a general scheme which will allow us to prove the existence of such currents and at the same time will provide a method to construct these currents. The fact that the generalized Toda system under investigation admits a conformal system of conserved higher-spin currents is not surprising of course since the analogous property is well-known for the ordinary Toda lattice field theory [4]- [6]. However an advantage of the method described in the next section is that it can be practically useful for the derivation of the explicit form of particular currents.

\section{Governing Equation}

Let us now introduce the equation that governs the structure of all the higher spin currents. In order to do that it is convenient to introduce generalized currents $J^{s}(\phi, \mu)$ which depend on a new parameter $\mu \in A q(2 ; 2 \nu)$ such that $\Pi_{-} \mu=\mu$, i.e. $\mu$ is an arbitrary lowest weight vector of the $s l_{2}$ algebra of the form

$$
\mu=\sum_{n=-\infty}^{\infty} \sum_{m=0}^{\infty} z^{n}\left(T^{-}\right)^{m} \mu_{n, m}
$$

where $\mu_{n, m}$ are arbitrary coefficients. The $\mu$ - independent part of the generalized currents $J^{s}(\phi, 0)$ will be identified with the higher spin currents in (5.46). The governing equation has the form

$$
\mathbf{D}_{\xi} J^{s}=0
$$

where

$$
\mathbf{D}_{\xi}=\left\langle\xi, \frac{\delta}{\delta \phi^{\prime}}\right\rangle-\left\langle\Pi_{-} U \xi, \frac{\delta}{\delta \mu}\right\rangle,
$$

$\xi$ is an arbitrary grade zero element of $A q(2 ; 2 \nu)$,

$$
\begin{gathered}
U=(1-D t)^{-1} \\
D(X)=\partial_{z}(X)+\left[\phi^{\prime}, X\right]+[\mu, X]
\end{gathered}
$$

and the variational derivatives are defined in the natural way as

$$
\left\langle\xi, \frac{\delta}{\delta \phi^{\prime}}\right\rangle \phi^{\prime}=\Pi_{0}(\xi) ; \quad\left\langle\eta, \frac{\delta}{\delta \mu}\right\rangle \mu=\Pi_{-}(\eta)
$$

For future use we also introduce

$$
\begin{gathered}
V=(1-t D)^{-1}, \\
G=t U=V t .
\end{gathered}
$$

The latter equation can be easily derived by expanding (6.4) and (6.7) in power series, as well as

$$
U-1=D G
$$

and

$$
V-1=G D
$$


Another useful formula is

$$
\delta(U D)=\delta(D V)=U \delta(D) V,
$$

where we have taken into account that $t$ is field independent.

As shown below, the variational equations (6.2) are indeed consistent in the following standard sense:

$$
\mathbf{D}_{\xi_{1}} \mathbf{D}_{\xi_{2}}=\mathbf{D}_{\xi_{2}} \mathbf{D}_{\xi_{1}} .
$$

If we rewrite (6.2) symbolically as

$$
\xi^{i} \frac{\partial}{\partial x^{i}} J=\xi^{i} A_{i}(J),
$$

where $x_{i}$ stands for $\phi^{\prime}$ while

$$
\xi^{i} A_{i}=\left\langle\Pi_{-} U \xi, \frac{\delta}{\delta \mu}\right\rangle
$$

is the second term of the linear operator on the left hand side of (6.2), then equation (6.12) is equivalent to the following zero-curvature conditions

$$
\frac{\partial}{\partial x^{i}} A_{j}-\frac{\partial}{\partial x^{j}} A_{i}=\left[A_{i}, A_{j}\right] .
$$

By using (6.14) the explicit form of this condition is

$$
Y=\left\langle\xi_{1}, \frac{\delta}{\delta \phi^{\prime}}\right\rangle \eta_{2}-\left\langle\eta_{1}, \frac{\delta}{\delta \mu}\right\rangle \eta_{2}-(1 \leftrightarrow 2)=0,
$$

where

$$
\eta_{i}=\Pi_{-} U \xi_{i} \quad i=1,2 .
$$

Let us now prove that $Y=0$. Using the explicit form of the operator $U$ one transforms $Y$ to the following form

$$
Y=\Pi_{-} U\left(\left[\xi_{1}, G \xi_{2}\right]-\left[\Pi_{-} U \xi_{1}, G \xi_{2}\right]\right)-(1 \leftrightarrow 2) .
$$

Now we use the identity (A.8) proven in Appendix A for the first term in the above equation to arrive at the following expression

$$
\begin{aligned}
Y & =\left(\Pi_{-}\left(\left[U \xi_{1}, G \xi_{2}\right]+D G\left(\left[\Pi_{-} U \xi_{1}, G \xi_{2}\right]\right)\right)\right. \\
& \left.-\Pi_{-} U\left[\Pi_{-} U \xi_{1}, G \xi_{2}\right]\right)-(1 \leftrightarrow 2),
\end{aligned}
$$

where the last term in (A.8) trivializes since $\xi_{i}$ is grade zero. The crucial point is then that the first term in the above equation can be rewritten as

$$
\begin{aligned}
\Pi_{-}\left[U \xi_{1}, t U \xi_{2}\right] & -(1 \leftrightarrow 2)=\left(\frac{1}{2} \Pi_{-}\left[T^{+},\left[t U \xi_{1}, t U \xi_{2}\right]\right]\right. \\
& \left.+\Pi_{-}\left[\Pi_{-} U \xi_{1}, t U \xi_{2}\right]\right)-(1 \leftrightarrow 2) .
\end{aligned}
$$


The first term in this expression is zero since $\Pi_{-}\left(\left[T^{+}, X\right]\right)=0$ for any $X$ due to (5.24). Inserting the remaining term in (6.20) into (6.19) and taking into account (6.9) we find that the terms cancel pairwise. We have thus proven that the governing equation (6.2) is indeed consistent.

From the general representation (6.15) one can write down a general solution for $J$ in the form

$$
J=P \exp \left[\int_{0}^{1} d s x^{i} A_{i}(s x)\right] j,
$$

where $j$ is an arbitrary $x$ - independent quantity and the $P$ ordering is with respect to $s$.

In order to implement this formula for the $A_{i}$ of (6.14) one has just to replace the parameter $\xi$ in (6.14) by $\phi^{\prime}$. This leads to the result

$$
J(\phi, \mu)=P \exp \left[\int_{0}^{1} d s\left\langle\Pi_{-} U\left(s \phi^{\prime}\right)\left(\phi^{\prime}\right), \frac{\delta}{\delta \mu}\right\rangle\right] j(\mu),
$$

where $j(\mu)$ is an arbitrary complex valued functional of $\mu$.

The main properties of this current which we will prove in Appendix B can be summarized as follows:

(i) The $\mu$-independent part $J(\phi, 0)$ of the current 6.22 is conserved according to the Hamiltonian equations of the Toda system for any $\phi$ - independent and time independent functional $j(\mu)$. The functional $j(\mu)$ parametrizes the full space of conserved currents. In what follows we refer to the functional $j(\mu)$ as the $j$-parameter.

(ii) From (6.22) it follows that

$$
j(\mu)=J(0, \mu)
$$

(iii) Since the governing equation (6.2)) is a first-order variational equation, according to the standard Leibniz rule, a product $J_{1 \times 2}=J_{1} J_{2}$ satisfies the governing equation if each of $J_{1}$ and $J_{2}$ do. From (6.23) it follows then that

$$
j_{1 \times 2}(\mu)=j_{1}(\mu) j_{2}(\mu) .
$$

This extends to any function $f(J)$ which can be represented as a power series. The $j$-parameter is then represented by $f(j)$.

(iv) Given two currents $J_{1}$ and $J_{2}$ satisfying the governing equation (6.2), the bilinear combination

$$
J_{1,2}(\phi, \mu)=\left\langle\frac{\delta J_{1}}{\delta \mu}, U D\left(\frac{\delta J_{2}}{\delta \mu}\right)\right\rangle
$$

satisfies the governing equation (6.2) too.

(v) The Poisson bracket for the two conserved currents with respect the standard Poisson structure of $\phi$ variables (5.44) is indeed a current of the form $J_{1,2}$, i.e.

$$
\left.\left\{J_{1}, J_{2}\right\}_{\phi}\right|_{\mu=0}=\left.J_{1,2}\right|_{\mu=0} .
$$

According to (iv) this shows that, as expected, the Poisson bracket of two conserved currents gives a new conserved current and $J_{1,2}$ gives an appropriate solution of the governing equation that corresponds to this conserved current. 
(vi) According to (6.25) the $j$-parameter for the conserved current $\left.\left\{J_{1}, J_{2}\right\}_{\phi}\right|_{\mu=0}$ therefore is

$$
j_{1,2}(\mu)=\left\langle\frac{\delta j_{1}}{\delta \mu}, U_{0} D_{0}\left(\frac{\delta j_{2}}{\delta \mu}\right)\right\rangle
$$

with

$$
\begin{gathered}
U_{0}=\left(1-D_{0} t\right)^{-1}, \quad V_{0}=\left(1-t D_{0}\right)^{-1}, \\
D_{0}(A)=\partial_{z}(A)+[\mu, A] .
\end{gathered}
$$

The right hand side of (6.27) defines a Poisson structure in the space of $\mu$ variables

$$
\left\{j_{1}(\mu), j_{2}(\mu)\right\}_{\mu}=\left\langle\frac{\delta j_{1}}{\delta \mu}, U_{0} D_{0}\left(\frac{\delta j_{2}}{\delta \mu}\right)\right\rangle
$$

The fact that the Jacobi identities are satisfied for this Poisson structure follows directly from the uniqueness of the solution of the governing equation and the fact that the original $\{,\}_{\phi}$ bracket respects the Jacobi identities. Nevertheless we will give an independent proof of the Jacobi identities for (6.30) in Appendix B.

The propositions (ii), and (iii) are straightforward and follow from the arguments of the text. The proofs of the propositions (i), (iv)-(vi) are given in Appendix B.

Note that the description of the currents in terms of a lowest weight vector field $\mu(z)$ is a different representation as compared to the zero-grade field $\phi(z)$. It is the Poisson brackets (6.30) between the $j$-parameters from which one can directly read off the algebra of conserved currents in the Toda system. Since the Poisson structure (6.30) is non-linear in $\mu$, from $(6.26)$ it follows that this non-linearity just reproduces a non-linear structure of the form of the Poisson algebra of conserved currents. The two representations are dual to each other in a certain sense. In the $\phi$ - representation the Poisson structure is simple but the form of the currents is quite complex. In the $\mu$-representation the Poisson structure on the other hand is nonlinear but the conserved currents are very simple. We will illustrate this in specific examples later.

Let us now discuss the relation between the generalized current (6.22) and the higher spin currents discussed in the previous section. From the property (iii) it follows that in order to construct a complete set of conserved currents it is enough to consider $j$ parameters linear in $\mu$.

We first note that the current (6.22) satisfies the following formula

$$
\begin{aligned}
J(\phi, \mu) & =J(0, \mu)+\int_{0}^{1} d s\left\langle\Pi_{-} U\left(s \phi^{\prime}\right)\left(\phi^{\prime}\right), \frac{\delta}{\delta \mu}\right\rangle P \exp \left[\int_{0}^{s} d s^{\prime}\left\langle\Pi_{-} U\left(s^{\prime} \phi^{\prime}\right)\left(\phi^{\prime}\right), \frac{\delta}{\delta \mu}\right\rangle\right] j(\mu) \\
& =j(\mu)+\int_{0}^{1} d s\left\langle\Pi_{-} U\left(s \phi^{\prime}\right)\left(\phi^{\prime}\right), \frac{\delta}{\delta \mu}\right\rangle J(s \phi, \mu) .
\end{aligned}
$$

The first equality in (6.31) can be obtained from (6.22) by using a power series expansion for a path ordered exponential. The second equality follows from a rescaling of the integration parameter $s^{\prime}$. The physical currents are identified with the (conserved) $\mu$ independent part of $J(\phi, \mu)$. From (6.31) it follows that

$$
J(\phi, 0)=j(0)+\int_{0}^{1} d s\left\langle\Pi \_\mathcal{U}\left(s \phi^{\prime}\right)\left(\phi^{\prime}\right), \epsilon_{+}\left(s \phi^{\prime}\right)\right\rangle,
$$


where

$$
\epsilon_{+}(\phi)=\left.\frac{\delta}{\delta \mu} J(\phi, \mu)\right|_{\mu=0}
$$

and

$$
\mathcal{U}=(1-\mathcal{D} t)^{-1}, \quad \mathcal{D}=\partial_{z}+\left[\phi^{\prime}, \quad\right] .
$$

Note that $\epsilon_{+}$is a highest weight vector since $\mu$ is a lowest weight vector. The effect of $\Pi_{-}$in (6.32) is unity because $\Pi_{+}\left(\epsilon_{+}\right)=\epsilon_{+}$. Furthermore the operator $\mathcal{U}$ can be partially integrated so that one is left with

$$
J(\phi, 0)=j(0)+\int_{0}^{1} d s\left\langle\phi^{\prime}, \mathcal{V}\left(s \phi^{\prime}\right) \epsilon_{+}\left(s \phi^{\prime}\right)\right\rangle
$$

where

$$
\mathcal{V}=(1-t \mathcal{D})^{-1}
$$

This equation is in fact the equation (5.48) with the solution for

$$
\epsilon^{s, 0}=\Pi_{0}\left(\mathcal{V}\left(\phi^{\prime}\right) \epsilon_{+}\left(\phi^{\prime}\right)\right)
$$

from the equation (5.27). Note that the constant $j(0)$ which appears in (6.35) does not affect the transformation properties. As is shown below this constant is related to the classical central charge in the model.

\section{Some Particular Currents from the Governing Equa- tion}

Let us illustrate how one can apply the governing equation in practical calculations. As a simplest example we consider the case of the spin - 1 current. In this case the $j$ - parameter has the form

$$
j(\mu)=\langle v, \mu\rangle,
$$

where the $c$-number parameter $v=v(z)$ is arbitrary. Note that only the zero grading part of $\mu$ is important. Inserting this formula into (6.22) and considering the $\mu$ - independent part we arrive at

$$
J^{1}(\phi, 0)=\left.\int_{0}^{1} d s\left\langle\Pi_{-} \mathcal{U}\left(s \phi^{\prime}\right)\left(\phi^{\prime}\right), \frac{\delta}{\delta \mu}\right\rangle\langle v, \mu\rangle\right|_{\mu=0}=\int_{0}^{1} d s\left\langle v, \mathcal{U}\left(s \phi^{\prime}\right)\left(\phi^{\prime}\right)\right\rangle=\int d z v(z) \operatorname{str}\left(\phi^{\prime}\right) .
$$

This current is indeed trivial since the mode $\operatorname{str}\left(\phi^{\prime}\right)$ decouples from the other fields in all expressions. It hence must have trivial Poisson brackets to all other currents of the theory.

Next we apply the formulae for the case of the conformal transformations. In this case the $j$ - parameter has the form

$$
j(\mu)=\left\langle u T^{+}, \mu\right\rangle,
$$

where $u=u(z)$ is an arbitrary conformal parameter and $\mu=\mu(z)$ is an arbitrary grade -1 lowest weight variable. (Let us remind ourselves that the definition (5.40) involves an 
integration over z.) Inserting this formula into $(6.22)$ and considering the $\mu$ - independent part we arrive at

$$
J^{2}(\phi, 0)=\left.\int_{0}^{1} d s\left\langle\Pi_{-} \mathcal{U}\left(s \phi^{\prime}\right)\left(\phi^{\prime}\right), \frac{\delta}{\delta \mu}\right\rangle\left\langle u T^{+}, \mu\right\rangle\right|_{\mu=0}=\int_{0}^{1} d s\left\langle u T^{+}, \mathcal{U}\left(s \phi^{\prime}\right)\left(\phi^{\prime}\right)\right\rangle .
$$

We notice that we only need to consider the linear term in the exponential because all the higher order terms will necessarily contain higher negative gradings which cannot contribute under the supertrace in the case under consideration where $T^{+}$is the only operator with positive grading. Similarly we only need to consider the second term in the expansion of $\mathcal{U}$. A straightforward calculations then leads to the following expression for the current

$$
J^{2}(\phi, 0)=-\int d z \operatorname{str}\left(\frac{1}{2} u(z)\left(\phi^{\prime}\right)^{2}+u^{\prime}(z) T^{0} \phi^{\prime}\right),
$$

which we recognize as the current leading to the transformation (3.21).

The natural conjecture for the spin - $s$ current is

$$
j_{v}^{s}(\mu)=\left\langle v\left(T^{+}\right)^{s-1}, \mu\right\rangle
$$

where $v=v(z)$ is an arbitrary parameter. Analogously one can use the formula (6.22) along with the property (i) for the derivation of the explicit form of the higher-spin currents. We hope to describe some more examples elsewhere. Here let us just note that from (6.22) it follows that only a finite number of terms is contributing for any fixed spin. The reason is that each term in the power series expansion of (6.22) contains the negatively graded operator $t$ so that only a finite number of terms can match a limited positive grade in the original spin- $s j$-parameter (7.6).

As an alternative to checking the algebra of the currents we can equally well do it for the $j$ - parameters using (6.30). As an example, let us check the conformal algebra in these terms.

$$
\left\{j_{1}(\mu), j_{2}(\mu)\right\}_{\mu}=\left\langle u_{1} T^{+}, U_{0} D_{0}\left(u_{2} T^{+}\right)\right\rangle=\left\langle u_{1} T^{+},\left(D_{0} t+D_{0} t D_{0} t\right)\left(u_{2}^{\prime} T^{+}\right)+\left(1+D_{0} t\right) u_{2}\left[\mu, T_{+}\right]\right\rangle,
$$

where again only the first few terms in the expansion of $U_{0}$ contribute. After some algebra one finds

$$
\left\{j_{1}(\mu), j_{2}(\mu)\right\}_{\mu}=\int d z\left(u_{1}^{\prime} u_{2}-u_{2}^{\prime} u_{1}\right) \operatorname{str}\left(T^{+} \mu(z)\right)+\frac{1}{2} \int d z u_{1} u_{2}^{\prime \prime \prime} \operatorname{str}\left(T^{+} T^{-}\right) .
$$

Reminding ourselves that we can project out with $P^{ \pm}$we can compute the final supertrace to obtain

$$
\left\{j_{1}(\mu), j_{2}(\mu)\right\}_{\mu}=j_{1,2}+\frac{1}{32}\left(1-4 \nu^{2}\right)\left(1 \mp \frac{2 \nu}{3}\right) \int d z u_{1} u_{2}^{\prime \prime \prime}
$$

with the convention that the upper (lower) sign corresponds to the $P^{+}\left(P^{-}\right)$sector and $j_{1,2}$ is the spin two current with the parameter $u_{1,2}=u_{1}^{\prime} u_{2}-u_{2}^{\prime} u_{1}$.

Let us now verify that the spin-s current (7.6) indeed transforms as a primary spin $s$ field under the conformal transformations.

$$
\left\{j_{u}^{2}(\mu), j_{v}^{s}(\mu)\right\}_{\mu}=\left\langle u T^{+}, U_{0} D_{0}\left(v\left(T^{+}\right)^{s-1}\right)\right\rangle=\left\langle u T^{+}, \sum_{n=0}^{\infty}\left(D_{0}\left(t D_{0}\right)^{n}\right)\left(v\left(T^{+}\right)^{s-1}\right)\right\rangle .
$$


By partial integration of $D_{0}$ and by using (5.23) one gets

$$
\left\{j_{u}^{2}(\mu), j_{v}^{s}(\mu)\right\}_{\mu}=-\left\langle u^{\prime} T^{+}, \sum_{n=1}^{\infty}\left(t\left(D_{0} t\right)^{n-1} D_{0}\right)\left(v\left(T^{+}\right)^{s-1}\right)\right\rangle-\left\langle u \tilde{\mu}, \sum_{n=1}^{\infty}\left(D_{0}\left(t D_{0}\right)^{n-1}\right)\left(v\left(T^{+}\right)^{s-1}\right)\right\rangle,
$$

where $\tilde{\mu}=\left(1-\Pi_{+}\right) \mu$, which implies that the zero grade part of $\mu$ is cancelled. In the first term on the right hand side of this equation we use the skewsymmetric property (5.22) along with the fact that $t\left(T^{+}\right)=-T^{0}$ and integrate $D_{0}$ by parts. In the second term we just integrate $D_{0}$ by parts. As a result we obtain

$$
\begin{aligned}
\left\{j_{u}^{2}(\mu), j_{v}^{s}(\mu)\right\}_{\mu} & =\left\langle u^{\prime \prime} T^{0}, \sum_{n=1}^{\infty}\left(\left(t D_{0}\right)^{n-1}\right)\left(v\left(T^{+}\right)^{s-1}\right)\right\rangle-\left\langle u^{\prime}\left[T^{0}, \mu\right], \sum_{n=1}^{\infty}\left(\left(t D_{0}\right)^{n-1}\right)\left(v\left(T^{+}\right)^{s-1}\right)\right\rangle \\
& +\left\langle(u \tilde{\mu})^{\prime}, \sum_{n=1}^{\infty}\left(t D_{0}\right)^{n-1}\left(v\left(T^{+}\right)^{s-1}\right)\right\rangle .
\end{aligned}
$$

Let us consider the first term on the right hand side of this equation. First one observes that the term with $n=1$ vanishes identically because of the properties of the supertrace. For the remaining terms we again use the skewsymmetric property of $t$ and the fact that $t\left(T^{0}\right)=-\frac{1}{2} T^{-}$to rewrite them as

$$
\frac{1}{2}\left\langle u^{\prime \prime} T^{-}, \sum_{n=2}^{\infty} D_{0}\left(t D_{0}\right)^{n-2}\left(v\left(T^{+}\right)^{s-1}\right)\right\rangle=\frac{1}{2} \delta(s-2) \int d z u v^{\prime \prime \prime} \operatorname{str}\left(T^{+} T^{-}\right),
$$

which we recognize as the central charge in the conformal algebra (7.8). To derive this result we used the fact that the terms with $t$ on the left hand side of (7.13) do not contribute since $\mu$ commutes with $T^{-}$and $\operatorname{str}\left(T^{-} t(A)\right)=0$ for any $A \in A q(2 ; 2 \nu)$.

For the second term in $(7.12)$ we first observe that the commutator $\left[T^{0}, \mu\right]$ is a lowest weight vector. Hence again all terms containing a $t$ vanish and one is left with

$$
-\left\langle u^{\prime}\left[T^{0}, \mu\right], \sum_{n=1}^{\infty}\left(\left(t D_{0}\right)^{n-1}\right)\left(v\left(T^{+}\right)^{s-1}\right)\right\rangle=(s-1)\left\langle u^{\prime} \mu,\left(v\left(T^{+}\right)^{s-1}\right)\right\rangle .
$$

Finally a similar analysis for the third term leads to

$$
\left\langle(u \tilde{\mu})^{\prime}, \sum_{n=1}^{\infty}\left(t D_{0}\right)^{n-1}\left(v\left(T^{+}\right)^{s-1}\right)\right\rangle=-\left\langle(u \tilde{\mu}),\left(v^{\prime}\left(T^{+}\right)^{s-1}\right)\right\rangle .
$$

Collecting these three terms together we get the standard result in the form (7.6)

$$
\left\{j_{u}^{2}(\mu), j_{v}^{s}(\mu)\right\}_{\mu}=\int d z\left((s-1) u^{\prime} v-v^{\prime} u\right) \operatorname{str}\left(\left(T^{+}\right)^{s-1} \tilde{\mu}(z)\right)+\frac{1}{2} \delta(s-2) \int d z u v^{\prime \prime \prime} s \operatorname{tr}\left(T^{+} T^{-}\right) .
$$

The appearance of $\tilde{\mu}$ in this formula has the effect that in the case of the spin - 1 current it indeed has a vanishing contribution. One can also directly check that the spin -1 current has the following Poisson brackets to any spin - $s$ current:

$$
\left\{j_{u}^{1}(\mu), j_{v}^{s}(\mu)\right\}_{\mu}=\delta(s-1) \int d z u v^{\prime} \operatorname{str}(1)
$$


i.e. it decouples from all $s \geq 2$ currents and has only a central charge contribution in its Poisson bracket with itself.

With similar techniques one can in principle compute any Poisson bracket between higher-spin currents. It is known that the full algebrae of higher-spin currents in the Toda systems are non-linear [4]-[6], [19]. In accordance with the property (iii) of the governing equation these non-linearities are manifested as non-linearities in $\mu$ since the individual higher-spin currents exhaust all linear functions of $\mu$. In fact this scheme can be used in an efficient way to compute $W_{n}$ algebrae which can be obtained by specifying $2 \nu=2 n \pm 1(\operatorname{cf}(2.24))$. In these cases all spins higher than $n$ decouple because of the properties of the supertrace.

Taking into account that according to (7.6) $s-1$ coincides with the negative grade of $\mu$ one can see that any two $j$ parameters $j_{1}(\mu)$ and $j_{2}(\mu)$ with spins $s_{1}$ and $s_{2}$, respectively, have some polynomial Poisson bracket (6.27). The reason is that each term involving $\mu$ in $D_{0}$ will contain an operator $t$ which decreases the grading by one and, therefore, an order of nonlinearity turns out to be limited by $\min \left(s_{1}, s_{2}\right)-1$. Let us note however that there is some difference between the form of the algebra $\tilde{W}_{1+\infty}$ (i.e. the second Gelfand-Dickey Hamiltonian structure) discussed in [19], which is at most bilinear in fields, and the form of the algebra (6.27) in which the degree of nonlinearity seems to increase with spin. We hope to analyze the relationship between the two algebras in more details elsewhere.

\section{Conclusions}

The construction of generalized Toda field theories performed in Section 3 can be extended also to the $b(\sigma)$ and $c(\sigma)$ cases by applying the truncations introduced at the end of Section 2 .

The example of the Toda field theory considered in this paper demonstrates that the infinite-dimensional higher-spin algebras applied originally to the analysis of the higherspin gauge dynamics in two, three and four space-time dimensions and underlying the model under consideration can be considered as a natural generalization of the finitedimensional matrix algebras. An interesting problem therefore is to extend the area of applicability of such an approach to a broader class of models. For example one can think of the generalized matrix models, etc. An interesting problem is to analyze if it is possible to extend the formalism described in this paper to the Affine Toda theories too.

At the moment we know that there exists a natural extension of our work to the supersymmetric case which we will discuss in a future publication. Of key interest is of course also to study the quantization of these models.

\section{Acknowledgments}

MV is grateful to O.V. Ogievetsky and M.V. Saveliev for stimulating discussions. LB would like to acknowledge the hospitality of the Lebedev Physical Institute of the Russian Academy of Sciences. MV acknowledges that the research described in this publication was made possible in part by Grant \# MQM300 from the International Science Foundation, grant 93-02-15541 from the Russian Basic Research Foundation, and grant 
94-2317 from INTAS. MV also would like to thank the Chalmers University for hospitality and The Royal Swedish Academy of Sciences for the financial support.

\section{Appendix A}

\section{Useful Identities}

The proof of integrability of higher-spin currents given in Appendix B is based on the following important identity

$$
\begin{aligned}
& G(A G(B)+(G(A)) B)-G(A) G(B) \\
= & G\left(\left(\Pi_{-} U(A)\right) G(B)+G(A)\left(\Pi_{-} U(B)\right)-V\left(\Pi_{+}((G(A)) G(B))\right)\right.
\end{aligned}
$$

valid for any two elements $A$ and $B$ of any arbitrary associative algebra which admits two derivations $D$ and $T$, i.e. $D(A B)=D(A) B+A D(B)$ and $T(A B)=T(A) B+A T(B)$. It is also assumed that there exists a linear mapping $t$ such that

$$
T t=1-\Pi_{-}, \quad t T=1-\Pi_{+}
$$

with some operators $\Pi_{ \pm}$. The operators $U$ and $G$ are defined in (6.4) and (6.8).

In applications to the Toda system under consideration we use the realization (6.5) for $D$ and $\left[T^{+}, \quad\right]$ for $T$.

Let us now prove the identity (A.1). Consider the quantity $X$

$$
X=G(T((G(A))(G(B)))) \text {. }
$$

By virtue of $(6.8)$ and $(5.23)$ one gets

$$
X=V\left(\left(1-\Pi_{+}\right)((G(A)) G(B))\right) .
$$

On the other hand applying $T$ to the right in (A.3) and using that $T$ is a differentiation of the algebra one finds

$$
X=G\left\{\left(\left(1-\Pi_{-}\right)(U(A))\right)(G(B))+(G(A))\left(\left(1-\Pi_{-}\right) U(B)\right)\right\} .
$$

As a result one arrives at

$$
\begin{aligned}
& G\{(U(A))(G(B))+(G(A))(U(B))\}-V(((G(A)) G(B)) \\
= & G\left\{\Pi_{-}((U(A)) G(B))+(G(A)) \Pi_{-}(U(B))\right\}-V\left(\Pi_{+}((G(A)) G(B))\right) .
\end{aligned}
$$

By replacing $U$ by $(U-1)+1=D G+1$ and $V$ by $(V-1)+1=G D+1$ with the aid of (6.9) and (6.10) one transforms the left hand side of (A.6) to

$$
\begin{gathered}
G\{(D G(A)) G(B)+(G(A))(D G(B))\}-G D((G(A)) G(B))+ \\
G(A(G(B))+(G(A)) B)-(G(A)) G(B) .
\end{gathered}
$$


Finally one observes that since $D$ is a differentiation the first three terms in the above expression cancel so that one is left with the identity (A.1).

Let us note that the identity (A.1) and the relation (6.9) allow us to identify the following useful new identity

$$
\begin{gathered}
U(A G(B)+G(A) B)=U(A) G(B)+G(A) U(B) \\
+\quad(U-1)\left(\Pi_{-} U(A) G(B)+G(A) \Pi_{-} U(B)\right)-D V \Pi_{+}(G(A) G(B)) .
\end{gathered}
$$

When the algebra admits a trace operation $\langle A\rangle$ which induces a bilinear form $\langle A, B\rangle=$ $\langle A B\rangle$ such that the operators $D, T$ and $t$ are skewsymmetric, i.e.

$$
\langle D(A)\rangle=\langle T(A)\rangle=0, \quad\langle A t(B)\rangle=-\langle t(A) B\rangle
$$

for arbitrary elements $A$ and $B$, the relation (A.1) leads to the following one

$$
\left\langle A G(B) G(C)-\Pi_{-}(U(A)) G(B) G(C)\right\rangle+\text { et cycl. }=0 .
$$

Another useful formula which holds for an arbitrary element $C$ and elements $A$ and $B$ obeying conditions

$$
[A, B]=0, \quad T(A)=T(B)=0
$$

(these conditions hold e.g. for highest weight vector elements of $A q(2 ; 2 \nu)$ ) is

$$
\begin{aligned}
\langle([V(A), V(B)] C\rangle & =\left\langle[V(A), V(B)] \Pi_{-} U(C)\right. \\
& \left.+V(A)\left[\Pi_{-} U D(B), G(C)\right]-V(B)\left[\Pi_{-} U D(A), G(C)\right]\right\rangle .
\end{aligned}
$$

The proof of this identity is rather technical. By virtue of (6.10) one gets

$$
\langle[V(A), V(B)] C\rangle=\langle[G D(A), G D(B)] C+([G D(A), B]+[A, G D(B)]) C\rangle .
$$

Applying (A.10) to the first term on the right-hand-side of this relation one arrives at:

$$
\begin{aligned}
\langle[V(A), V(B)] C\rangle & =\langle-[D(A), G D(B)] G(C)-[G D(A), D(B)] G(C) \\
& +[G D(A), G D(B)] \Pi_{-} U(C) \\
& +\left(\left[\Pi_{-} U D(A), G D(B)\right]+\left[G D(A), \Pi_{-} U D(B)\right]\right) G(C) \\
& +([G D(A), B]+[A, G D(B)]) C\rangle .
\end{aligned}
$$

Now using the simple fact that $D$ is a derivation one transforms (A.14) to

$$
\begin{aligned}
\langle[V(A), V(B)] C\rangle & =\langle-D([A, G D(B)] G(C)+[G D(A), B] G(C)) \\
& +([A, D G D(B)] G(C)+[A, G D(B)] D G(C)-A \leftrightarrow B) \\
& +[G D(A), G D(B)] \Pi_{-} U(C) \\
& +\left(\left[\Pi_{-} U D(A), G D(B)\right]+\left[G D(A), \Pi_{-} U D(B)\right]\right) G(C) \\
& +([G D(A), B]+[A, G D(B)]) C\rangle .
\end{aligned}
$$


Using (6.8), (A.2) and ( $\mathrm{A.9})$ one transforms the second line in the above relation to get

$$
\begin{aligned}
\langle[V(A), V(B)] C\rangle & =\langle(A T([G D(B), G(C)])-A[D(B), G(C)]-A[G D(B), C] \\
& \left.\left.+A\left[\Pi_{-} U D(B), G(C)\right]\right)+A\left[G D(B), \Pi_{-} U(C)\right]-A \leftrightarrow B\right) \\
& +[G D(A), G D(B)] \Pi_{-} U(C) \\
& +\left(\left[\Pi_{-} U D(A), G D(B)\right]+\left[G D(A), \Pi_{-} U D(B)\right]\right) G(C) \\
& +([G D(A), B]+[A, G D(B)]) C\rangle .
\end{aligned}
$$

Now one observes that the term involving $T$ vanishes because of (A.11) while all terms which do not involve $\Pi_{-}$cancel pairwise (taking into account that $[D(A), B]+[A, D(B)]=$ 0 due to (A.11)). The remaining terms can be easily summed up to the right hand side of (A.12).

In practical calculations it is often useful to use a power series expansion for the formulae (A.1) and (A.12) which arises from the geometric progression formula for $U$ and $V$ in (6.4) and (6.7).

\section{Appendix B}

\section{Proofs of the Properties of the Generalized Currents}

In this Appendix we prove the properties (i)-(vi) of the generalized currents formulated in Section 6.

Proof for Proposition (i):

A straightforward calculation shows

$$
\left.\{\mathcal{H}, J\}_{\phi}\right|_{\mu=0}=\left.\left\langle T^{+},\left[H^{-}, \frac{\delta J}{\delta \phi^{\prime}}\right]\right\rangle\right|_{\mu=0}=-\left.\left\langle H^{-},\left[T^{+}, \frac{\delta J}{\delta \phi^{\prime}}\right]\right\rangle\right|_{\mu=0} .
$$

By virtue of the governing equation (6.2) we rewrite this expression as

$$
\left.\left\langle\eta, \frac{\delta J}{\delta \mu}\right\rangle\right|_{\mu=0}
$$

with

$$
\eta=-\left.\Pi_{-} U\left(\left[T^{+}, H^{-}\right]\right)\right|_{\mu=0} .
$$

Next we use (6.9) and (5.23) to rewrite $\eta$ as

$$
\eta=-\Pi_{-}\left(\left[T^{+}, H^{-}\right]+\mathcal{U}\left(\mathcal{D}\left(1-\Pi_{+}\right) H^{-}\right)\right) .
$$

One then observes that $\mathcal{D} H^{-} \equiv 0\left(\mathcal{D} H^{-}=R_{+-}^{-} \equiv 0\right.$ due to the definition of the Lorentz connection in the original zero curvature approach $), \Pi_{+} H^{-}=0$ and $\Pi_{-}\left(\left[T^{+}, H^{-}\right]\right)=0$. This concludes the proof of Proposition (i).

The proofs of the Propositions (ii) and (iii) are obvious.

Proof for Proposition (iv): 
In order to prove that $\mathbf{D}_{\xi}\left(\left\langle\frac{\delta J_{1}}{\delta \mu}, U D\left(\frac{\delta J_{2}}{\delta \mu}\right)\right\rangle\right)=0$ provided that $\mathbf{D}_{\xi} J_{1}=\mathbf{D}_{\xi} J_{2}=0$ consider the following expression

$$
X=\mathbf{D}_{\xi}\left(\left\langle\frac{\delta J_{2}}{\delta \mu}, U D\left(\frac{\delta J_{1}}{\delta \mu}\right)\right\rangle\right)-\left\langle\frac{\delta \mathbf{D}_{\xi}\left(J_{2}\right)}{\delta \mu}, U D\left(\frac{\delta J_{1}}{\delta \mu}\right)\right\rangle-\left\langle\frac{\delta J_{2}}{\delta \mu}, U D\left(\frac{\delta \mathbf{D}_{\xi}\left(J_{1}\right)}{\delta \mu}\right)\right\rangle .
$$

Using the explicit forms of the $\mathbf{D}_{\xi}$ and $D$ one finds

$$
X=\left\langle\frac{\delta J_{2}}{\delta \mu}, U\left[\xi-\Pi_{-} U(\xi), V\left(\frac{\delta J_{1}}{\delta \mu}\right)\right]\right\rangle+\left(\left\langle\frac{\delta J_{2}}{\delta \mu}, U\left[\Pi_{-} U D \frac{\delta J_{1}}{\delta \mu}, t U(\xi)\right]\right\rangle-(1 \leftrightarrow 2)\right) .
$$

By identifying $\frac{\delta J_{2}}{\delta \mu}, \frac{\delta J_{1}}{\delta \mu}$ and $\xi$ with $A, B$, and $C$ respectively one concludes that $X=0$ as a consequence of the identity (A.12). This concludes the proof of Proposition (iv).

Let us now explain why proposition (v) is true. From the definition of the Poisson structure (5.45) one gets

$$
\left\{J_{1}, J_{2}\right\}_{\phi}=\left\langle\frac{\delta J_{1}}{\delta \phi^{\prime}}, \partial_{z} \frac{\delta J_{2}}{\delta \phi^{\prime}}\right\rangle
$$

By virtue of the governing equation (6.2) one finds

$$
\left\{J_{1}, J_{2}\right\}_{\phi}=\left\langle\frac{\delta J_{1}}{\delta \mu}, U \partial_{z} \frac{\delta J_{2}}{\delta \phi^{\prime}}\right\rangle .
$$

Using the governing equation a second time one gets

$$
\left\{J_{1}, J_{2}\right\}_{\phi}=-\left\langle\frac{\delta J_{2}}{\delta \mu}, U \Pi_{0} \partial_{z} V \frac{\delta J_{1}}{\delta \mu}\right\rangle .
$$

The crucial observation now is that when $\mu=0$ one can first replace $\partial_{z}$ by $\mathcal{D}$ in the above formula (because of the operator $\Pi_{0}$, the term in $\mathcal{D}$ which involves the commutator vanishes since $\phi^{\prime}$ commutes with neutral elements) and, second, use that

$$
\left.\left\langle\rho_{1}, U \Pi_{0} \partial_{z} V \rho_{2}\right\rangle\right|_{\mu=0}=\left.\left\langle\rho_{1}, U D \rho_{2}\right\rangle\right|_{\mu=0}
$$

for arbitrary positive graded $\rho_{1,2}$ because $\left.D\right|_{\mu=0}=\mathcal{D}$ has grading zero while $t$ has grading -1 , so that the left hand side just accounts for all possibilities for insertions of the projection operator $\Pi_{0}$ inside those terms on the right hand side which have a nonvanishing supertrace. This completes the proof of Proposition (iv).

To prove the proposition (vi) one observes that it follows from (6.27) that

$$
\begin{aligned}
X_{1,2,3} & =\left\{\left\{j_{1}, j_{2}\right\}_{\mu}, j_{3}\right\}_{\mu}+\text { et cycl. }=\left\langle V_{0}\left(A_{1}\right),\left[\Pi_{-}\left(D_{0} V_{0}\left(A_{3}\right)\right), V_{0}\left(A_{2}\right)\right]\right\rangle+\text { et cycl. } \\
& =-\left\langle\left[V_{0}\left(A_{1}\right), V_{0}\left(A_{2}\right)\right], \Pi_{-} D_{0} V_{0}\left(A_{3}\right)\right\rangle+\text { et cycl. }
\end{aligned}
$$

where we use the notation $A_{i}=\frac{\delta j_{i}}{\delta \mu}$ so that $A_{i}$ are highest weight vectors obeying the properties (A.11). With the aid of the substitution (6.10) one gets

$$
\begin{aligned}
X_{1,2,3} & =-\left\langle\left[G_{0} D_{0}\left(A_{1}\right), G_{0} D_{0}\left(A_{2}\right)\right] \Pi_{-} D_{0} V_{0}\left(A_{3}\right)\right\rangle \\
& -\left\langle\left(\left[A_{1}, G_{0} D_{0}\left(A_{2}\right)\right]+\left[G_{0} D_{0}\left(A_{1}\right), A_{2}\right]\right) \Pi_{-} D_{0} V_{0}\left(A_{3}\right)\right\rangle+\text { et cycl. }
\end{aligned}
$$


Then, using the basic identity (A.10) for the first term on the right hand side of (B.5) one finds

$$
\begin{aligned}
X_{1,2,3} & =-\left\langle D_{0}\left(A_{1}\right)\left[G_{0} D_{0}\left(A_{2}\right), G_{0} D_{0}\left(A_{3}\right)\right]\right\rangle \\
& -\left\langle\left(\left[A_{1}, G_{0} D_{0}\left(A_{2}\right)\right]+\left[G_{0} D_{0}\left(A_{1}\right), A_{2}\right]\right) \Pi_{-} D_{0} V_{0}\left(A_{3}\right)\right\rangle+\text { et cycl. . }
\end{aligned}
$$

Using (6.10) once more along with the cyclic property of the supertrace and the fact that $\left[A_{i}, A_{j}\right]=0$ and therefore $\left[D A_{i}, A_{j}\right]+\left[A_{i}, D A_{j}\right]=0$ one finds

$$
\begin{aligned}
X_{1,2,3}= & -\left\langle D_{0}\left(A_{1}\right)\left[V_{0}\left(A_{2}\right), V_{0}\left(A_{3}\right)\right]\right\rangle \\
& -\left\langle\left(\left[A_{1}, V_{0}\left(A_{2}\right)\right]+\left[V_{0}\left(A_{1}\right), A_{2}\right]\right) \Pi_{-} D_{0} V_{0}\left(A_{3}\right)\right\rangle+\text { et cycl. } .
\end{aligned}
$$

By an integration by parts

$$
\begin{aligned}
X_{1,2,3} & =\left\langle A_{1}\left(\left[D_{0} V_{0}\left(A_{2}\right), V_{0}\left(A_{3}\right)\right]+\left[V_{0}\left(A_{2}\right), D_{0} V_{0}\left(A_{3}\right)\right]\right\rangle\right. \\
& -\left\langle\left(\left[A_{1}, V_{0}\left(A_{2}\right)\right]+\left[V_{0}\left(A_{1}\right), A_{2}\right]\right) \Pi_{-} D_{0} V_{0}\left(A_{3}\right)\right\rangle+\text { et cycl. } .
\end{aligned}
$$

Next we use the following identity

$$
\begin{aligned}
& 0=\left\langle A_{1},\left[T^{+},\left[V_{0}\left(A_{2}\right), V_{0}\left(A_{3}\right)\right]\right]\right\rangle+\text { et cycl. } \\
& =\left\langle A_{1}\left(\left[\left(1-\Pi_{-}\right) D_{0} V_{0}\left(A_{2}\right), V_{0}\left(A_{3}\right)\right]+\left[V_{0}\left(A_{2}\right),\left(1-\Pi_{-}\right) D_{0} V_{0}\left(A_{3}\right)\right]\right\rangle+\right.\text { et cycl.,(B. }
\end{aligned}
$$

which is a consequence of $\left\langle\left[A_{i},\left[T^{+}, B\right]\right]\right\rangle=\left\langle\left[\left[A_{i}, T^{+}\right], B\right]\right\rangle=0$ and (5.23), (6.8). Finally one observes that the right-hand side of $(\mathbb{B} .8)$ exactly coincides with the expression on the right-hand side of $(\mathbb{B . 9})$. This completes the proof that $X_{1,2,3}=0$. 


\section{Appendix $\mathrm{C}$}

\section{Notations and Definitions}

$$
\begin{aligned}
& T^{0}=\frac{1}{4}\left\{a^{-}, a^{+}\right\}, \quad T^{ \pm}=\frac{1}{2}\left(a^{ \pm}\right)^{2}, \\
& {\left[T^{0}, T^{ \pm}\right]= \pm T^{ \pm}, \quad\left[T^{-}, T^{+}\right]=2 T^{0},} \\
& \left.E_{n, m}=\left(\left(a^{+}\right)^{n}\left(a^{-}\right)^{m}\right)_{W} \equiv \frac{1}{(n+m) !}\left(\left(a^{+}\right)^{n}\left(a^{-}\right)^{m}+((n+m) !-1)\right) \text { permutations }\right), \\
& h_{n}=E_{n, n}, \\
& \operatorname{str}\left(P^{ \pm} E_{n, m} E_{m, n}\right)=(-1)^{n} n ! m ! \beta^{ \pm}(n+m), \\
& \partial_{ \pm}=\frac{1}{\sqrt{2}}\left(\partial_{0} \pm \partial_{1}\right) \\
& \mathcal{D} A=\partial_{+} A+\left[\partial_{+} \phi, A\right] \\
& t\left(E_{n, m}\right)=-\frac{1}{m+1} E_{n-1, m+1} \quad n>0 ; \quad t\left(E_{0, m}\right)=0 \text {, } \\
& {\left[T^{+}, t(x)\right]=x-\Pi_{-}(x), \quad t\left(\left[T^{+}, x\right]\right)=x-\Pi_{+}(x),} \\
& \Pi_{-}\left(E_{0, m}\right)=E_{0, m} ; \quad \Pi_{-}\left(E_{n, m}\right)=0 \quad n>0 \text {, } \\
& \Pi_{+}\left(E_{n, 0}\right)=E_{n, 0} ; \quad \Pi_{+}\left(E_{n, m}\right)=0 \quad m>0, \\
& \Pi_{0}\left(E_{n, n}\right)=E_{n, n} \equiv h_{n} ; \quad \Pi_{0}\left(E_{n, m}\right)=0 \quad n \neq m, \\
& \langle\xi, \phi\rangle=\int d z \operatorname{str} \xi(z) \phi(z), \\
& \phi^{\prime}=\partial_{+} \phi \text {. } \\
& \left\langle\xi^{\prime}, \eta\right\rangle+\left\langle\xi, \eta^{\prime}\right\rangle=0 \\
& \left\{\langle\xi, \phi\rangle,\left\langle\eta, \phi^{\prime}\right\rangle\right\}=\langle\xi, \eta\rangle, \\
& \mathbf{D}_{\xi}=\left\langle\xi, \frac{\delta}{\delta \phi^{\prime}}\right\rangle-\left\langle\Pi_{-} U \xi, \frac{\delta}{\delta \mu}\right\rangle \text {, } \\
& U=(1-D t)^{-1} \\
& D(X)=\partial_{z}(X)+\left[\phi^{\prime}, X\right]+[\mu, X] \\
& \left\langle\xi, \frac{\delta}{\delta \phi^{\prime}}\right\rangle \phi^{\prime}=\Pi_{0}(\xi) ; \quad\left\langle\eta, \frac{\delta}{\delta \mu}\right\rangle \mu=\Pi_{-}(\eta) . \\
& V=(1-t D)^{-1} \text {, } \\
& G=t U=V t \text {. } \\
& j_{1,2}(\mu)=\left\langle\frac{\delta j_{1}}{\delta \mu}, U_{0} D_{0}\left(\frac{\delta j_{2}}{\delta \mu}\right)\right\rangle, \\
& U_{0}=\left(1-D_{0} t\right)^{-1}, \quad V_{0}=\left(1-t D_{0}\right)^{-1}, \\
& \mathcal{U}=(1-\mathcal{D} t)^{-1}, \quad \mathcal{D}=\partial_{z}+\left[\phi^{\prime}, \quad\right], \\
& \mathcal{V}=(1-t \mathcal{D})^{-1} \text {. }
\end{aligned}
$$




\section{References}

[1] A. N. Leznov and M.V. Saveliev, Lett. Math. Phys. 3 (1979) 489.

[2] A. V. Mikhailov, JETP Lett. 30 (1979) 443.

[3] D.I. Olive and N. Turok, Nucl. Phys. B220 [FS8] (1983) 491; Nucl. Phys. B257 [FS14] (1985) 277.

[4] A. Bilal and J-L. Gervais, Phys. Lett. 206 (1988) 412.

[5] T. Takebe, Comm. Math. Phys. 129 (1990) 281.

[6] J. Balog, L. Feher, L. O'Raifeartaigh, P. Forgacs and A. Wipf, Annals of Physics 203 (1990) 76-136.

[7] A.N. Leznov and M.V. Saveliev, Group Theoretical Methods for Integration of Nonlinear Dynamical Systems, Progress in Physics, v. 15, Birkhäuser-Verlag, Basel, 1992.

[8] A. V. Mikhailov, M. A. Olshanetsky and A.M. Perelomov, Comm. Math. Phys. 79 (1981) 473.

[9] O. I. Bogoyavlensky, Izv. Acad. Nauk SSSR. Ser. Mat. 51 (1987) 737;

I. Bakas, Comm. Math. Phys. 134 (1990) 487;

Q-Han Park, Phys. Lett. 236B (1990) 429;

M.V. Saveliev, Teor. Mat. Fiz. 92 (1992) 457.

[10] M.A. Vasiliev, JETP Lett. 50 (1989) N8, 374; Int. J. Mod. Phys. A6 (1991) 1115.

[11] C.N. Pope, L.J. Romans and X. Shen, Phys. Lett. B236 (1990) 173; Phys. Lett. B242 (1990) 401.

[12] M.A. Vasiliev, Preprint FIAN/TD/15-95; in preparation.

[13] M.A. Vasiliev, Class. Quant. Grav. 11 (1994) 649.

[14] M.A. Vasiliev, Mod. Phys. Lett. A7 (1992) 3689.

[15] M.A. Vasiliev, Phys. Lett. B285 (1992) 225.

[16] K. Aoki and E.D'Hoker, Nucl. Phys. B387 (1992) 576.

[17] G.L. Rcheulishvili and M.V. Saveliev, Phys. Lett. A130 (1988) 69.

[18] E.G.B. Hohler and K. Olaussen, Int. J. Mod. Phys. A10 (1995) 687.

[19] I. Bakas and E. Kiritsis, Int. J. Mod. Phys. A7 Suppl. 1A (1992) 55;

F. Yu and Y.-S. Wu, Nucl. Phys. B373 (1992) 713. 
[20] E.P. Wigner, Phys. Rev. 77 (1950) 711;

D. G. Boulware and S. Deser, Il Nouvo Cimento, XXX (1963) 231;

N. Mukunda, E.C.G. Sudarshan, J.K. Sharma and C.L. Mehta, J. Math. Phys. 21 (1980) 2386.

[21] E. Bergshoeff B. de Wit, and M.A. Vasiliev, Nucl. Phys. B366 (1990) 315.

[22] E. Bergshoeff, M.P. Blencowe and K.S. Stelle, Commun. Math. Phys. 128 (1990) 213 ;

M. Bordemann, J.Hoppe and P. Schaller, Phys. Lett. B232 (1989) 199.

[23] E.S. Fradkin and M.A. Vasiliev, Dokl. Acad. Nauk. 29 (1986) 1100; Ann. of Phys. 177 (1987) 63; Phys. Lett. 189 (1987) 89; Nucl. Phys. 291 (1987) 141.

[24] R.S. Pierce, Associative Algebras, Springer-Verlag New York, 1982.

[25] M.V. Saveliev and A.M. Vershik, Commun. Math. Phys. 126 (1989) 367. 